\title{
Method of phase space beam dilution utilizing bounded chaos generated by rf phase modulation
}

\author{
Alfonse N. Pham ${ }^{*}$ and S. Y. Lee \\ Indiana University Center for Exploration of Energy and Matter, \\ Bloomington, Indiana 47408, USA \\ K. Y. $\mathrm{Ng}^{\ddagger}$ \\ Fermi National Accelerator Laboratory, Batavia, Illinois 60510, USA
}

(Received 24 April 2015; published 10 December 2015)

\begin{abstract}
This paper explores the physics of chaos in a localized phase-space region produced by rf phase modulation applied to a double rf system. The study can be exploited to produce rapid particle bunch broadening exhibiting longitudinal particle distribution uniformity. Hamiltonian models and particle-tracking simulations are introduced to understand the mechanism and applicability of controlled particle diffusion. When phase modulation is applied to the double rf system, regions of localized chaos are produced through the disruption and overlapping of parametric resonant islands and configured to be bounded by well-behaved invariant tori to prevent particle loss. The condition of chaoticity and the degree of particle dilution can be controlled by the rf parameters. The method has applications in alleviating adverse space-charge effects in high-intensity beams, particle bunch distribution uniformization, and industrial radiation-effects experiments.
\end{abstract}

DOI: 10.1103/PhysRevSTAB.18.124001

PACS numbers: 29.27.Bd, 29.27.Eg, 52.59.Sa

\section{INTRODUCTION}

There exist numerous techniques to instigate the blowup of longitudinal beam emittance. Each technique is tailored to address one or a combination of the following difficulties in the handling of high-intensity beams in a storage ring: severe effects of intrabeam scattering that leads to uncontrolled transverse emittance blowup, collective instabilities, large space-charge tune shifts resulting in possible encounters with lattice resonances that will limit the performance of a machine, etc. For applications in radiation-effects experiments at Indiana University (IU), a 20-m compact electron storage ring, designed with a pair of gradient damping wiggler magnets for momentum-compaction factor variability, is currently under construction [1]. The storage ring will serve as a debuncher and can be injected with $50-100 \mathrm{MeV}$ electrons from a linac to accumulate a bunch of total charge up to $600 \mathrm{nC}$. In the presence of radiation damping, the natural bunch length is of the order of $10-100$ ps. The radiation experiments require the enhancement of duty factor by broadening the electron bunch up to $40 \mathrm{~ns}$ in length with longitudinal particle distribution uniformity.

\footnotetext{
*alfonse@msu.edu

shylee@indiana.edu

ng@fnal.gov
}

Published by the American Physical Society under the terms of the Creative Commons Attribution 3.0 License. Further distribution of this work must maintain attribution to the author(s) and the published article's title, journal citation, and DOI.
Longitudinal broadening can be attained by employing a double rf potential with optimally flattened potential well and elongated rf bucket width [2,3], but this method provides insufficient broadening for an initially narrow bunch of the order of $100 \mathrm{ps}$. The use of barrier rf manipulation, wherein a set of rf potential barriers of opposing polarity configured on either end of the particle bunch are shifted adiabatically apart, can also provide bunch broadening [4]. Unfortunately, for compact ferrite-dominated rf systems that are often used due to space limitations, difficulties arise in establishing a barrier width with rise time less than $0.05 \mu$ s arise due to core losses in ferrite materials at higher frequencies. This renders the barrier rf technique unsuitable for many compact rings.

Phase modulation applied to a higher harmonic rf systems has been used for controlled longitudinal emittance blowup at various facilities [5]. At the CERN SPS, a bunch of full width $\sim 1.6 \mathrm{~ns}$ had been blown up to $\sim 3 \mathrm{~ns}$ before transferring to the LHC. The mechanism of the blowup is by rf phase modulation that drives the bunch partially into nearby parametric resonant islands. More recent methods of bunch broadening using band-limited phase noise are promising [6]. Bunch shaping by rf voltage modulation with band-limited white noise has been employed to produce linear density uniformity without blowing up the longitudinal emittance [7]. At the KEK PS, quadruple synchrotron oscillation $2 \omega_{s}$ was fed into the voltage control loop of the low-level rf feedback system together with white-noise band $\Delta \omega$ from a waveform generator, where $\omega_{s}$ is the small-amplitude synchrotron frequency and $\Delta \omega \ll \omega_{s}$. Particles near the bunch center will be driven into 2:1 resonances and move away from the bunch center, 


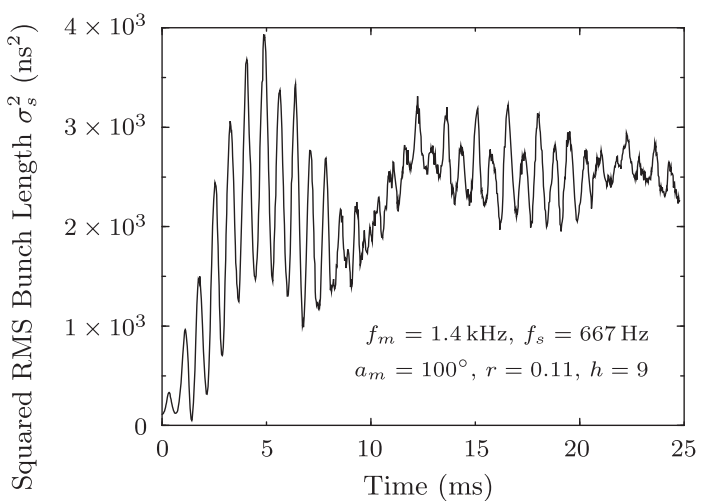

FIG. 1. Measurement of the squared rms bunch length $\sigma_{s}^{2}$ as a function of time where the phase difference between the two rf cavities was $\Delta \phi_{o} \sim 245^{\circ}$. The data were obtained by digitizing beam position monitor signals at $1 \mathrm{~ns}$ resolution where phase modulation of frequency $f_{m}=1.4 \mathrm{kHz}$ and amplitude $a_{m}=100^{\circ}$ was applied to the higher-frequency cavity of a double rf system with rf voltage ratio $r=0.11$ and harmonic ratio $h=9$. (The data is courtesy of D. Jeon et al.)

while particles near the edge having synchrotron frequency less than $\omega_{s}-\Delta \omega / 2$ will not be affected. This technique successfully yielded bunch density uniformity, but do not provide adequate bunch broadening required for the radiation-effects experiments.

Despite the aforementioned longitudinal emittance blowup techniques, the solutions we require are different. We wish to broaden the length of a radiation-damped electron bunch 400 - to 800 -fold, or the emittance $10^{5}$ - to $10^{6}$-fold, while maintaining bunch density uniformity. We suggest driving the bunch into large chaotic regions that are bounded by well-behaved tori. The diffusion of particles by phase modulation applied to a single cavity in a double if system has been observed experimentally at the Indiana University Cyclotron Facility (IUCF) cooler storage ring (CSR) in 1997 $[8,9]$. Figure 1 shows the square of the rms bunch length exhibiting linear growth as a function of time. More importantly, the experiments at the IUCF found the mechanism of diffusion to be highly sensitive to the phase difference $\Delta \phi_{o}$ between the two rf cavities. This motivates the prospect of bunch dilution where rf cavities are phase modulated for radiation-effects experiments, first reported in 2010 [10].

This paper has been organized in the following manner. In Sec. II, a theoretical model of the double rf system, study of the rf phase modulation parametric dependence, and discussion on expected resonances that are excited will be presented. In Sec. III, symplectic multiparticle simulations are used to produce Poincaré surfaces of section to verify the mechanisms discussed in Sec. II, and probe relevant rf parameters resulting in maximal bunch broadening exhibiting longitudinal particle distribution uniformity. In Sec. IV, the implementation of the phase-space dilution studies will be discussed. Finally, the conclusion and closing remarks will be given in Sec. V.

\section{THE DOUBLE RF MODEL}

A radiation-damped bunch is first prepared at the $\mathrm{rf}$ phase coordinate $\phi=0$ in longitudinal phase space, which is the origin of a stationary $\mathrm{rf}$ bucket produced by a primary rf cavity operating with rf harmonic number $h_{1}$ and $\mathrm{rf}$ voltage $V_{1}$. A secondary if cavity with harmonic number $h_{2}=h h_{1}$, voltage $V_{2}=r V_{1}$, and rf phase difference $\Delta \phi_{o}$ referenced to the primary cavity is then switched on nonadiabatically. Both the rf cavities will receive the applied rf phase modulation.

The particle motion in the longitudinal phase space with the secondary rf cavity activated is described by the Hamiltonian [11]

$$
H=H_{o}+H_{1} \text {. }
$$

Here,

$$
H_{o}=\frac{1}{2} \nu_{s} \delta^{2}+V(\phi)
$$

where $\nu_{s}$ is the small-amplitude synchrotron tune referenced to the primary rf cavity in the absence of the secondary rf cavity,

$$
\delta=\frac{h_{1} \eta_{c}}{\nu_{s}}\left(\frac{\Delta p}{p_{o}}\right)
$$

is the normalized fractional momentum deviation coordinate referenced to the primary rf cavity, with $\eta_{c}$ being the longitudinal phase-slip factor and $\Delta p=p-p_{o}$ the offmomentum coordinate referenced to an ideal particle of momentum $p_{o}$. The resulting double rf potential with both rf cavities activated is

$$
V(\phi)=\nu_{s}\left\{1-\cos \phi-\frac{r}{h}\left[1-\cos \left(h \phi+\Delta \phi_{o}\right)\right]\right\},
$$

where $\Delta \phi_{o}$ is the rf phase difference between the two rf waves. The modulation of the rf phases is given by

$$
H_{1}=\nu_{s} a_{m} \delta \sin \left(\nu_{m} \theta+\eta\right),
$$

where $\nu_{m}$ is the phase modulation tune, $a_{m}$ is the modulation amplitude, $\eta$ is the modulation phase, and $\theta$ serves as the independent time coordinate, which increases by $2 \pi$ per revolution. In the discussion below, $H_{0}$ and $H_{1}$ will be treated, respectively, as the unperturbed and perturbed portions of the Hamiltonian.

For the Hamiltonian approach to be valid, some assumptions have been made. First, the effects of radiation damping and quantum excitation in the bunch broadening process were not included. Bunch broadening is assumed to occur much faster than radiation damping effects. A discussion on the validity and limitation of this assumption will be given in Sec. III D. Second, since the momentum 
change of a particle is applied locally by the rf cavities, a turn-by-turn description of the longitudinal motion is more appropriate. Here, the Hamiltonian in Eqs. (1) and (5) has been constructed as a smooth approximation of the turn-byturn symplectic longitudinal mapping equations by introducing the continuous time variable $\theta$ in order to facilitate analysis [12]. A discussion concerning its validity will be given in Sec. III.

Since the equilibrium beam profile is dependent on the shape of the rf potential, in the absence of applied phase modulation $\left(H_{1}=0\right)$, the double rf system can be configured to flatten the potential well to provide larger bunching factors. When parameters are chosen to satisfy the condition where $r h=1$ and $\Delta \phi_{o}=0$, the double rf potential-well bottom will be optimally flat. This method alone will only broaden the bunch by a factor of about 2 . Instead, rf phase modulation will be introduced to initiate controlled diffusion so that the bunch can be broadened further to fill nearly three-quarters of the primary rf bucket. The equations of motion for particles in a double rf system with applied phase modulation are

$$
\begin{gathered}
\frac{d \phi}{d \theta}=\nu_{s}\left[\delta+a_{m} \sin \left(\nu_{m} \theta+\eta\right)\right], \\
\frac{d \delta}{d \theta}=-\nu_{s}\left[\sin \phi-r \sin \left(h \phi+\Delta \phi_{o}\right)\right],
\end{gathered}
$$

where Eq. (6) implies that the phases of both rf cavities are being simultaneously modulated in the model.

It has been shown that applied phase modulation will excite parametric resonances in a particle bunch [8]. When a multitude of parametric resonances are strongly driven and overlap, large regions of chaotic particle motion are generated [9]. In order to facilitate particle diffusion, the relative phase difference between the two rf cavities will need to deviate from zero, resulting in an asymmetric potential [10]. This will allow a small radiation-damped bunch that is strongly bounded at $\phi=0$ to diffuse outward to large regions of overlapping parametric resonances beyond. To avoid confusion, we emphasize that the rf phase coordinate $\phi$, and the location of phase-space origin ( $\phi=0$ and $\delta=0$ ) is always referenced to that of the stationary rf bucket when only the primary rf cavity is activated. For brevity, the phrase primary-rf-only longitudinal phase space will be used.

\section{A. Choice of phase difference parameter $\Delta \phi_{o}$}

The strengths of parametric resonances driven by rf phase modulation are functions of the action $J$ [8] [see Eq. (17) and Fig. 6]. For a given dynamical system with canonical coordinates $\phi$ and $\delta$, the expression for $J(E)$ obtained from the unperturbed Hamiltonian in Eq. (2) is given as

$$
J=\frac{1}{2 \pi} \oint \delta\left(\phi^{\prime}\right) d \phi^{\prime}=\frac{1}{2 \pi} \oint \sqrt{\frac{2}{\nu_{s}}[E-V(\phi)]} d \phi,
$$

and vanishes at the location of the minimum or bottom of the rf potential well. Up to a scale factor, $J$ represents the area in phase space enclosed by a torus of a given energy $E$ as shown in Fig. 3. For a conventional single rf system or double rf system operating in phase $\left(\Delta \phi_{o}=0\right)$, the minimum of the rf potential or stable fixed point of the rf bucket is located at the phase coordinate $\phi=0$. Since the bunch is initially located at $\phi=0$, where $J=0$, the resonance strengths due to phase modulation vanishes. As a result, the applied phase modulation is extremely ineffective at driving a small radiation-damped bunch away from the origin of the primary-rf-only phase space where it may utilize overlapping and collapsed resonant islands to drive particle diffusion. For this reason, the minimum of the $\mathrm{rf}$ potential must be shifted away from $\phi=0$ so that the action at the initial location of the radiation-damped bunch becomes optimally large. A double rf system with relative phase $\Delta \phi_{o} \neq 0$ and $\neq \pi$ will be necessary to accomplish this.

The location of the rf potential minimum $\phi_{o}$ is a function of the phase difference $\Delta \phi_{o}$ between the two rf waveforms, and is given by

$$
\left.\frac{d V(\phi)}{d \phi}\right|_{\phi=\phi_{o}}=\sin \phi_{o}-r \sin \left(h \phi_{o}+\Delta \phi_{o}\right)=0 .
$$

The maximum offset of the rf potential minimum $\phi_{o}\left(\Delta \phi_{o}\right)$ is given by $d \phi_{o} / d \Delta \phi_{o}=0$, and is therefore

$$
\phi_{o}= \pm \sin ^{-1} r .
$$

This occurs when the phase difference of the two rf cavities is configured to be

$$
\Delta \phi_{o}= \pm \frac{\pi}{2} \mp h \sin ^{-1} r .
$$

We will restrict the discussion for the case where $r h=1$. For $r=1 / 2$ and $h=2$, the largest offset of the potential minimum from the origin of the primary-rf-only longitudinal phase space was found to be $\phi_{o}= \pm 30^{\circ}$, corresponding to a phase difference between the two rf cavities of $\Delta \phi_{o}= \pm 30^{\circ}$. The dependence of the potential-minimum offset $\phi_{o}$ as a function of the phase difference parameter $\Delta \phi_{o}$ is shown in Fig. 2 where several shapes of the rf potential and the corresponding potential minima are depicted. Figure 3 shows the shape of the potential where $\Delta \phi_{o}=30^{\circ}$ with corresponding phase-space torus of motion about the stable fixed point at $\phi_{o}=\pi / 6$ and the associated energy levels where $r=1 / 2$ and $h=2$ have been chosen. Augmenting the rf potential will enable the action $J$, and thus the strengths of the driven parametric 

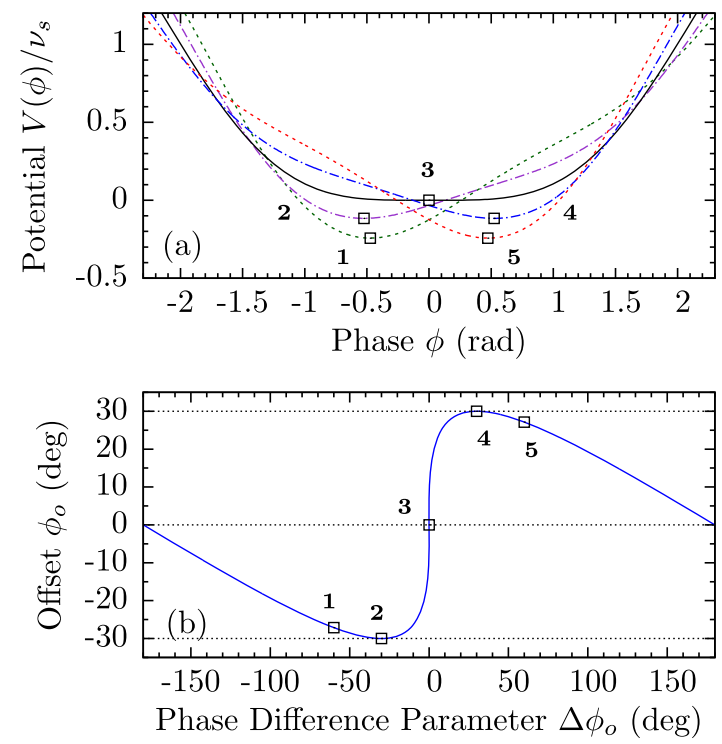

FIG. 2. (a) rf potential $V(\phi)$ as a function of longitudinal phase $\phi$ for $r=1 / 2$ and $h=2$. The boxes denote each respective potential minimum where the $\mathrm{rf}$ phase difference parameters are (1) $\Delta \phi_{o}=-60^{\circ}$, (2) $-30^{\circ}$, (3) $0^{\circ}$, (4) $30^{\circ}$, and (5) $60^{\circ}$. (b) The resulting potential-minimum offset $\phi_{o}$ with varying $\Delta \phi_{o}$.

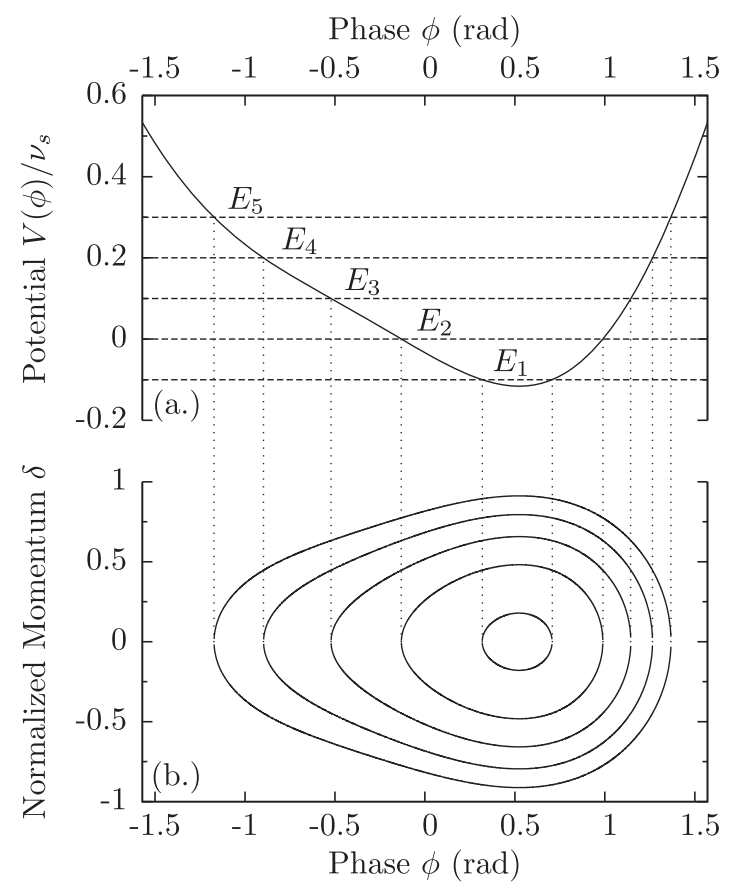

FIG. 3. (a) rf potential $V(\phi)$ as a function of longitudinal phase $\phi$ for $r=1 / 2, h=2$, and $\Delta \phi_{o}=30^{\circ}$. The particle energy levels $E_{1}=-0.1, E_{2}=0.0, E_{3}=0.1, E_{4}=0.2$, and $E_{5}=0.3$ are shown corresponding to (b) each respective torus of motion in phase space.

resonances at the initial location of the particle bunch, to be nonvanishing. Under these conditions, particles will be able to be driven to regions of thick overlapping resonances beyond.
Simulation results described in Sec. III have been performed for all values of $\Delta \phi_{o}$ varied by small steps. It shows that diffusion only occurs in the range $20^{\circ} \lesssim$ $\left|\Delta \phi_{o}\right| \lesssim 55^{\circ}$. Thus rf phase difference parameters in the vicinity of $\Delta \phi_{o}=30^{\circ}$ will be the focus of this study. When $\Delta \phi_{o}=\pi$ or $\Delta \phi_{o}=0$, such as in conventional Landau cavities, simulations reveal that no large chaotic regions are generated by overlapping resonant island chains. Therefore large blowup of longitudinal beam emittance will not occur. Only a few-fold increase in emittance was observed as particles traveled into nonoverlapping resonant islands. This result was supported by experiments at the IUCF CSR [8,9], where a huge emittance increase was observed at some optimal rf phase difference $\Delta \phi_{o}$ as depicted in Fig. 1. The emittance increase was negligible when $\Delta \phi_{o}$ was chosen to be near zero or $\pi$.

We wish to point out that the perturbed Hamiltonian of Eq. (5) implies the phases of both rf systems are modulated with the same frequency and amplitude. An alternate method exists that applies phase modulation to only the secondary if system. This method has been explored at the IUCF CSR in 1997 [8,9]. Both methods indicate that the rf phase difference $\Delta \phi_{0}$ must be nonzero to generate overlapping resonant island chains that form large chaotic regions for particles diffusion. Here, we choose to investigate the method of modulating both rf cavities by the same modulation parameters as it simplifies analysis, although the alternate method might be easier to implement experimentally.

\section{B. Synchrotron tune and resonance strengths}

The unperturbed Hamiltonian in Eq. (2) is canonically transformed to the action-angle variables using the generating function of the second kind,

$$
F_{2}(\phi, J)=\int_{\phi_{1}}^{\phi} \delta\left(\phi^{\prime}\right) d \phi^{\prime}=\int_{\phi_{1}}^{\phi} \sqrt{\frac{2}{\nu_{s}}\left[E(J)-V\left(\phi^{\prime}\right)\right]} d \phi^{\prime},
$$

where the energy $E(J)$ is the inverse function of action $J(E)$ as defined in Eq. (8), while the conjugate angle variable is

$$
\psi=\frac{\partial F_{2}}{\partial J}=\frac{\partial E}{\partial J} \int_{\phi_{1}}^{\phi} \frac{\partial \delta\left(\phi^{\prime}\right)}{\partial E} d \phi^{\prime}=\frac{Q_{s}}{\nu_{s}} \int_{\phi_{1}}^{\phi} \frac{d \phi^{\prime}}{\delta\left(\phi^{\prime}\right)} .
$$

The $\phi_{1}$ in Eqs. (12) and (13) is a turning point of phase excursion in longitudinal phase space for a torus of motion that corresponds to action $J$ or energy $E(J)$, and $Q_{s}=$ $(\partial J / \partial E)^{-1}$ is the synchrotron tune. The angle variable $\psi$ in Eq. (13) advances by $\pi$ when $\phi$ reaches the opposing turning point of phase excursion where $\phi=\phi_{2}$. This gives rise to a method of calculating the synchrotron tune by way of an elliptic integral expression, 


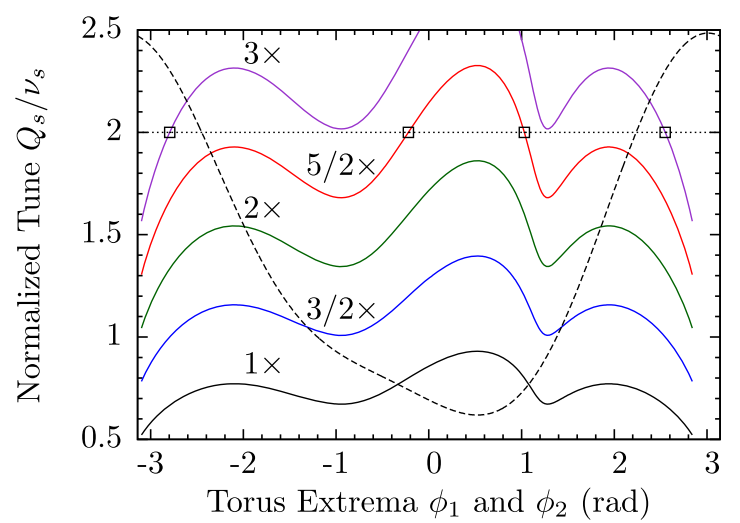

FIG. 4. Normalized synchrotron tune $Q_{s} / \nu_{s}$ as a function of torus extrema $\phi_{1}$ and $\phi_{2}$. The $\nu_{m} / \nu_{s}=2$ horizontal dotted-line intercepts depict the location of $n: M$ parametric resonances driven by phase modulation. The dashed line overlays the asymmetric shape of the potential $V(\phi) / \nu_{s}$ where the double rf parameters are $r=1 / 2, h=2$, and $\Delta \phi_{o}=30^{\circ}$.

$$
\frac{Q_{s}(J)}{\nu_{s}}=\pi\left(\int_{\phi_{1}}^{\phi_{2}} \frac{d \phi^{\prime}}{\sqrt{\frac{2}{\nu_{s}}\left[E(J)-V\left(\phi^{\prime}\right)\right]}}\right)^{-1} .
$$

The resulting normalized synchrotron tune, obtained by numerical integration, as a function of torus extrema is shown in Fig. 4, for the case where $r=1 / 2, h=2$, and $\Delta \phi_{o}=30^{\circ}$. The small-amplitude synchrotron tune is $Q_{s}=\nu_{s}\left(\cos \phi_{o}-h r \sqrt{1-\sin ^{2} \phi_{o} / r^{2}}\right)$. At the optimal potential-minimum offset, the small-amplitude synchrotron tune becomes $Q_{s}=\nu_{s} \sqrt{1-r^{2}}$, which is independent of the rf harmonic number ratio $h$. Parametric resonances are excited within the bunch by coherent kicks from the applied phase modulation that satisfies the resonant condition,

$$
n Q_{s}-M \nu_{m}=0
$$

where individually driven parametric resonances are identified using the notation $n: M$ resonance. The horizontal dotted line in Fig. 4 depicts the applied phase modulation where the modulation tune is $\nu_{m}=2 \nu_{s}$. The intercepts of the synchrotron tune curves with this dotted line reveal the locations of fixed points of the corresponding parametric resonant islands driven by the applied phase modulation. By varying $\nu_{m}$, resonances are excited at various locations in longitudinal phase space.

The synchrotron tune can also be computed as a function of the action $J$, which is related to the phase-space oscillation amplitude, as shown in Fig. 5. When $\nu_{m}=2 \nu_{s}$, the small bunch at the origin of the primaryrf-only phase space where $J=0.092$ will be driven strongly by the $5: 2$ resonance due to the close proximity of the initial bunch to the $5: 2$ resonant islands. The $3: 1$ resonance, being first order, is more strongly excited and will overlap with the $5: 2$ resonant island chain to create large

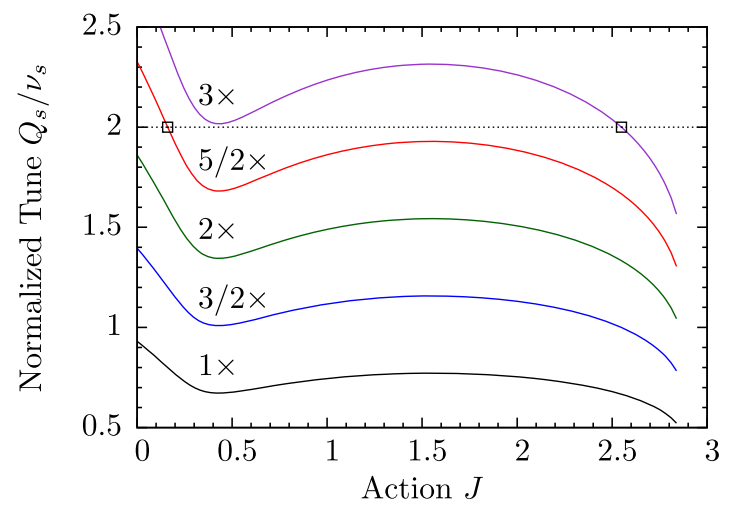

FIG. 5. Normalized synchrotron tune $Q_{s} / \nu_{s}$ as a function of the action $J$. The $\nu_{m} / \nu_{s}=2$ horizontal dotted-line intercepts depict the location of $n: M$ parametric resonances driven by phase modulation where the double rf parameters are $r=1 / 2, h=2$, and $\Delta \phi_{o}=30^{\circ}$. The initial location of the bunch at the origin of the primary-rf-only phase space corresponds to the action $J=0.092$.

chaotic regions at higher modulation amplitude. The analysis summarized in Figs. 4 and 5 will be used to predict the location various $n: M$ parametric resonances driven within a bunch. With the synchrotron tune being larger near the origin of the primary-rf-only phase space as compared to at the edges, this will ensure the condition where the generated central chaotic region will be bounded by well-behaved tori preventing particle loss.

The perturbed Hamiltonian can be expressed in terms of action-angle variables by expanding the fractional momentum deviation $\delta$ into a Fourier series,

$$
\delta(J, \psi)=\sum_{n=-\infty}^{+\infty} g_{n}(J) e^{i n \psi},
$$

where the resonance strength function is given as [13]

$$
g_{n}(J)=\frac{1}{2 \pi} \int_{-\pi}^{\pi} \delta e^{-i n \psi} d \psi
$$

The complex resonance-strength function can be expressed as $g_{n}(J)=\left|g_{n}(J)\right| e^{+i \chi_{n}}$ where $\chi_{n}$ denotes the phase. Using the fact that the complex Fourier coefficients are related by $g_{n}=g_{-n}^{*}$, the total Hamiltonian in terms of action angles can be expressed as

$$
\begin{aligned}
H= & H_{o}(J)+a_{m} \nu_{s}\left\{\sum _ { n = 1 } ^ { + \infty } | g _ { n } ( J ) | \left[\sin \left(\nu_{m} \theta+\eta+\chi_{n}+n \psi\right)\right.\right. \\
& \left.\left.+\sin \left(\nu_{m} \theta+\eta-\chi_{n}-n \psi\right)\right]+g_{o}(J) \sin \left(\nu_{m} \theta+\eta\right)\right\} .
\end{aligned}
$$

The Hamiltonian in Eq. (18) contains all the parametric resonances that are excited by the applied phase 


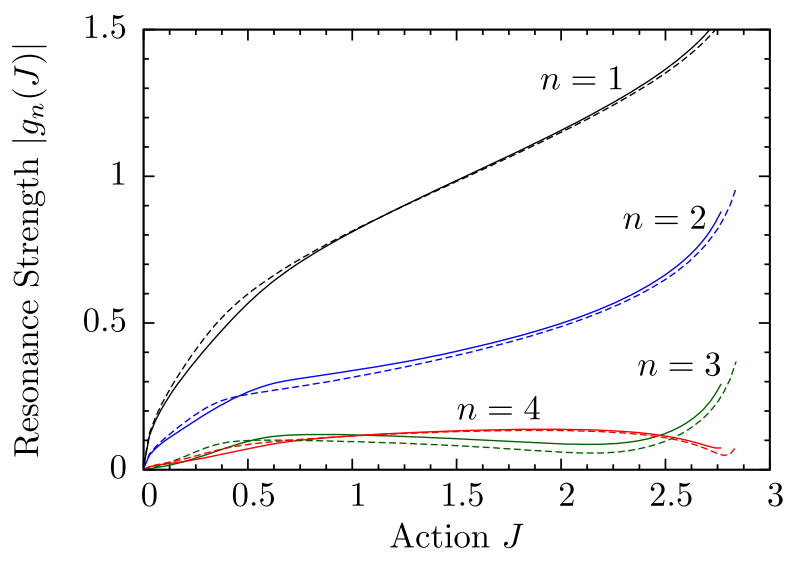

FIG. 6. Magnitude of the resonance strength $\left|g_{n}(J)\right|$ as functions of action $J$ for $n: 1$ parametric resonances where the double rf parameters are $r=1 / 2$ and $h=2$. The curves are in solid for $\Delta \phi_{o}=30^{\circ}$ and dashes for $\Delta \phi_{o}=45^{\circ}$.

modulation. The higher-order parametric resonances, i.e., $n: M$ resonances with $M>1$, are revealed by performing higher-order perturbations via $M$ canonical perturbation transformations from action-angle coordinates $(\psi, J)$ to the new coordinates $(\bar{\psi}, \bar{J})$. For example, the $5: 2$ resonance is produced by the combination of lower-order resonances, such as the $2: 1$ and $3: 1$ resonances through another canonical transformation [11]. The first-order perturbation terms are sinusoidal. The higher-order perturbations, however, will produce stationary terms ( $\theta$-independent). These terms, when added to the unperturbed Hamiltonian $H_{o}(J)$ will modify the $\mathrm{rf}$ potential. The potential-well minimum as well as the positions of the parametric resonances will be perturbed accordingly.

The calculations of the resonant strength $\left|g_{n}(J)\right|$ as functions of the action $J$ are shown in Fig. 6 for harmonics $n=1,2,3,4$ and order $M=1$, where the double if parameters are $r=1 / 2, h=2$, and $\Delta \phi_{o}=30^{\circ}$. The same calculation has been completed for $\Delta \phi_{o}=45^{\circ}$ shown in the dashes. The analysis shows that lower harmonic parametric resonances are, in general, more strongly driven than higher harmonics. Also lower-order resonances are more strongly driven than higher-order resonances. It is important to note that $\left|g_{n}(J)\right|$ does indeed vanish as the action approaches $J=0$ at the location of the $\mathrm{rf}$ potential minimum.

For sinusoidal phase modulations applied to a symmetric rf potential, only odd harmonic parametric resonances are generated. Due to the asymmetry of $V(\phi)$ resulting from the shifted potential-well minimum, $g_{n}(J)$ no longer vanishes for even $n$. This implies that both odd and even $n: 1$ parametric resonances can be simultaneously excited. Depending on the choice of $\nu_{m}$ and $a_{m}$ configured in the double rf system, both odd and even harmonic parametric resonances can be exploited to optimize the process of beam dilution.

\section{SIMULATION RESULTS}

Numerical simulations were carried out by tracking particles at each successive revolution from the $k$ th revolution to the $(k+1)$ th revolution according to [12]

$$
\begin{array}{r}
\phi_{k+1}=\phi_{k}+2 \pi \nu_{s}\left[\delta_{k}+a_{m} \sin \left(2 \pi k \nu_{m}+\eta\right)\right], \\
\delta_{k+1}=\delta_{k}-2 \pi \nu_{s}\left[\sin \phi_{k+1}-r \sin \left(h \phi_{k+1}+\Delta \phi_{o}\right)\right] .
\end{array}
$$

Since the rf cavities are localized rather than distributed continuous along the storage ring, these equations provide more accurate and realistic particle tracking than the equations of motion, Eqs. (6) and (7), with continuous time variable $\theta$. The particle tracking equations also assume that the primary and secondary rf cavities are situated at the same location. Thus Eq. (19) gives the longitudinal phase drift of particles from the exit of the rf cavities, around the storage ring, and back to the entrance of the rf cavities, and Eq. (20) gives the momentum delivered to particles while crossing the rf cavities that are infinitesimally thin. Simple modifications of these equations can be made to model two cavities that are located at different places along the storage ring. The resulting differences between the turn-by-turn tracking and tracking with continuous time is negligibly small, because the small-amplitude synchrotron tune $\nu_{s}$ in the presence of only the primary rf cavity is typically small. In the simulations presented here, $1 / \nu_{s}=1030$ was used. The symplecticity of the multiparticle tracking will be used to uncover resonance structures arising from the applied phase modulation and resonant-island topographies promoting particle diffusion bounded by invariant tori.

\section{A. rf phase difference $\Delta \phi_{o}=30^{\circ}$}

The Poincare surface of section for $\Delta \phi_{o}=30^{\circ}$ that gives rise to the largest potential-minimum offset from the origin of the primary-rf-only phase space will first be studied. Since the choice of modulation tune near $\nu_{m} \sim 2 \nu_{s}$ yields reasonable diffusion results, it will be used to illustrate resonant island structures resulting from increasing modulation amplitude $a_{m}$. A detailed discussion on the upper and lower limits for arbitrarily chosen $\nu_{m}$ and $a_{m}$ parameters for optimal phase-space dilution will be presented later in Sec. III B.

With phase modulation amplitude $a_{m}=8^{\circ}$, the Poincaré section in Fig. 7 was produced by stacking stroboscopic frames obtained from particle tracking, where the longitudinal motion of $1 \times 10^{4}$ macroparticles were evolved. To ensure that the phase-space diagrams remain stationary or nonrotating, stroboscopic frames are taken every modulation number $N_{m}=1 / \nu_{m}=515$ (for $\nu_{m} / \nu_{s}=2$ ), chosen to be exactly an integer revolution number within the simulations. These Poincaré surfaces of section eliminate uninteresting time dependencies due to synchrotron rotation and sinusoidal phase modulation in the longitudinal phase 


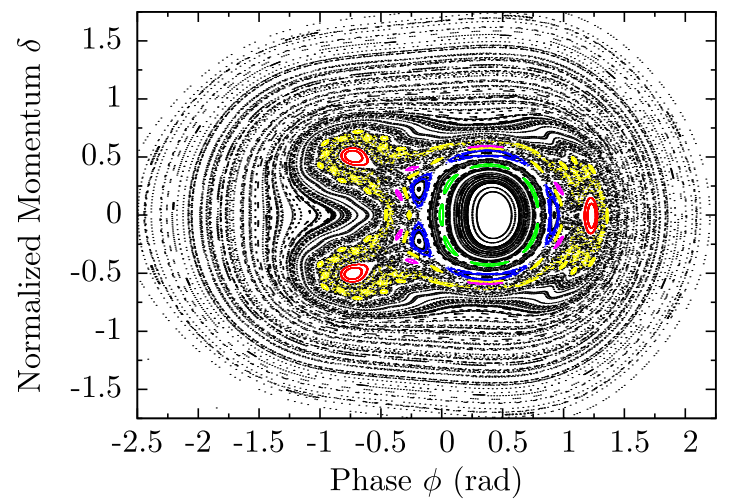

FIG. 7. Nondiffusive Poincaré surface of section with parameters $a_{m}=8^{\circ}$ and $\Delta \phi_{o}=30^{\circ}$. The initial particle bunch is confined between the (blue) $5: 2$ and (green) $7: 3$ resonant islands. The (magenta) 8:3 resonance can be seen about to merge with the layers of overlapping (yellow) higher-order resonances surrounding the resonant islands of the (red) $3: 1$ resonance. Well-behaved tori bounding the regions of chaotic particle motion are observed.

space, leaving behind only the resonant behavior for analysis.

Since particle diffusion has yet to occur at low $a_{m}$, an excessively large Gaussian-distributed particle bunch, with bunch sizes of the order of $\sigma_{\phi} \sim 1 \mathrm{rad}$ and $\sigma_{\delta} \sim 1$, was tracked to outline various relevant resonance structures resulting from the chosen parameters. The result depicted in Fig. 7 was for modulated phase $\eta=0$ [see Eq. (19)] which will be used for all the analyses presented in this section. It shows the 5:2, 7:3 and many higher-order resonances in the central region. Beyond that, remnants of the $8: 3$ resonance and higher-order resonances are partially merging to form chaotic layers surrounding the 3:1 resonant islands. At the edge of the phase space, strongly driven higher-order resonances form the well-behaved tori bounding the layers of chaotic particle motion that are driven by the applied phase modulation. In order for the particle bunch that is initially at the primary-rf-only phase-space origin to be enlarged by diffusion, the $5: 2,7: 3$, and other centrally bounding higher-order resonances must collapse and the central region of well-behaved tori shrink. This will allow the bunch to be driven out to the ever-growing chaotic region formed by the accumulation of collapsed and overlapping resonances within the well-behaved bounding tori. This condition has been found to start occurring when the modulation amplitude approaches $a_{m} \approx 49^{\circ}$ for the $\mathrm{rf}$ phase difference parameter $\Delta \phi_{o}=30^{\circ}$.

Now let us examine the effects of phase modulation on a Gaussian-distributed bunch of rms bunch length $\sigma_{\phi}=$ $1 \times 10^{-3} \mathrm{rad}$ and momentum spread $\sigma_{\delta}=1 \times 10^{-3}$ consisting of $1 \times 10^{4}$ macroparticles placed at the origin of the primary-rf-only phase space. The total bunch length of $\sim 100 \mathrm{ps}$ corresponds to $\pm 5 \sigma$ of the overall particle distribution. The Poincaré sections with $a_{m}=58^{\circ}$, shown in

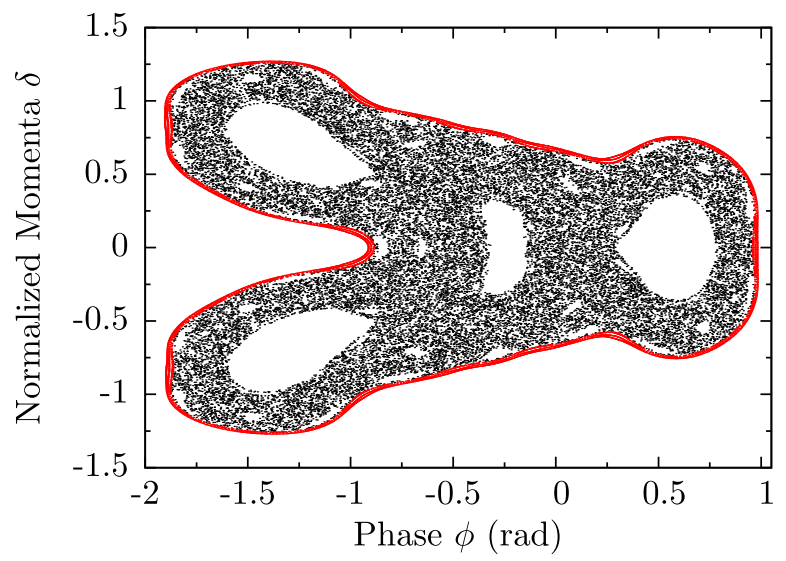

FIG. 8. Poincaré surface of section depicting the diffusion of the Gaussian bunch of $\sigma_{\phi}=1 \times 10^{-3} \mathrm{rad}$ and $\sigma_{\delta}=1 \times 10^{-3}$ initially at the primary-rf-only phase-space origin after 1.5 million revolutions where $a_{m}=58^{\circ}$ and $\Delta \phi_{o}=30^{\circ}$. The red points depict the single-particle tracking of test particles placed initially near the edge to show that the structure is indeed bounded.

Fig. 8, depict the last five modulation periods after tracking for 1.5 million revolutions where particle diffusion has taken place. The red points depict stable tori bounding the diffused chaotic region. The small bunch at the origin of the primary-rf-only phase space had been driven into the thick chaotic layers surrounding the separatrices of the $3: 1$ resonance. The thick stochastic layers, on the other hand, come from the overlapping of the $5: 2,7: 3,8: 3$, and possibly many other higher-order resonances. Aside from the four empty spaces corresponding to the four stable fixed-points associated with the $3: 1$ resonance, the resulting longitudinal distribution of particles in the bunch is nearly uniform. Particle tracking was continued by increasing the number of revolutions to be much greater than 1.5 million, to show that the particles filling the large chaotic region remained bounded and no particle loss was observed. The Poincaré section as well as the particle distribution uniformity were left unchanged and persisted independent of time after equilibration as shown in Fig. 8.

The rms beam sizes are computed turn by turn and shown in Fig. 9. The squares of the rms bunch sizes, $\sigma_{\phi}^{2}$ and $\sigma_{\delta}^{2}$, are shown to initially grow linearly with revolution number (time). This is an indication that the bunch broadening effect is a diffusion process. The growths of both $\sigma_{\phi}^{2}$ and $\sigma_{\delta}^{2}$ eventually level out and equilibrate after $\sim 1.5 \times 10^{5}$ revolutions. The rms bunch length and momentum spread were calculated to be $\sigma_{\phi}=0.81 \pm 0.03 \mathrm{rad}$ and $\sigma_{\delta}=$ $0.57 \pm 0.01$, respectively, after diffusion have equilibrated. Thus the bunch has been broadened roughly 800 -fold.

Next we will examine how the small radiation-damped bunch at the origin of the primary-rf-only phase space gradually broadened to become the distribution shown in Fig. 8. The evolution of particle diffusion in phase space is shown in Fig. 10. Particles are observed to be smoothly 


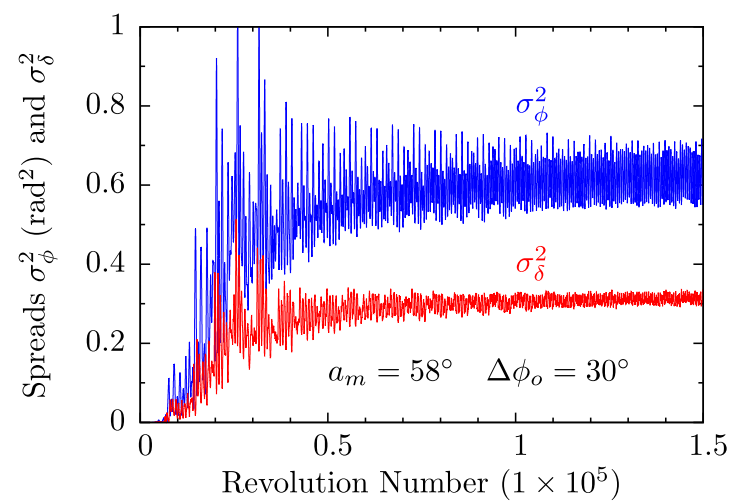

FIG. 9. Squared of the longitudinal bunch sizes as functions of revolution number for $a_{m}=58^{\circ}$ and $\Delta \phi_{o}=30^{\circ}$ as the diffusion process evolves. The evolution starts out linearly with time and equilibrates after about $1.5 \times 10^{5}$ revolutions.

streaming from the primary-rf-only phase-space origin to the $3: 1$ resonant islands rather than hopping from one island to the another, and gradually filling up the large bounded stochastic region. This is another indication that process is a diffusion process. The boundary of red points in the last frame in Fig. 10 was obtained by placing single particles initially at positions approaching the bounding tori then tracked for 1.5 million revolutions. The innermost torus of red points constitutes the boundary of a chain of overlapping resonant islands that forms the well-behaved tori, confirming that the diffusion of particles will be bounded.

When the modulation amplitude is increased from $a_{m} \approx 49^{\circ}$ to $73^{\circ}$, the Poincaré sections remained virtually unchanged, with rms bunch length and momentum spread calculated after diffusion staying fairly constant at $\sigma_{\phi} \approx 0.8 \mathrm{rad}$ and $\sigma_{\delta} \approx 0.58$, respectively, as shown in Fig. 11. The only significant change to be noted is the gradual shifting of the entire phase-space structure to the left with respect to the origin of the primary-rf-only phase space. This can be readily observed by noting the displacement of the central region in Fig. 7 as compared to Fig. 8. This shift would be to the right instead if stroboscopic frames were observed at the modulation phase of $\eta=\pi$ in Eq. (19). The gradual shift is a consequence of the movement of the rf potential-well bottom and the positions of the parametric resonances as the modulation amplitude $a_{m}$ increases. When one of the three 3:1 resonant islands has been shifted to include the primary-rf-only phase-space origin where the bunch is originally located, no diffusion will occur. This is exactly what was observed in the hatched region between $75^{\circ} \lesssim a_{m} \lesssim 85^{\circ}$ in Fig. 11.

When the modulation amplitude was increased past $a_{m} \approx 115^{\circ}$, rapid particle loss was observed. The nondiffusive Poincaré section when $a_{m}=104^{\circ}$, shown in Fig. 12, depicts a Poincaré section riddled with many collapsing higher-order resonances as the phase-space structure is on the verge of particle loss. The chains of resonant islands at the edge, associated with many strongly driven resonances that once formed the well-behaved bounding tori, are showing signs of overlap and disruption. This will leave the phase-space structure unbounded and susceptible to uncontrolled longitudinal beam blowup.

\section{B. Choice of modulation tune and amplitude}

Longitudinal particle-tracking simulations were used to map the modulation fraction $\nu_{m} / \nu_{s}$ and modulation

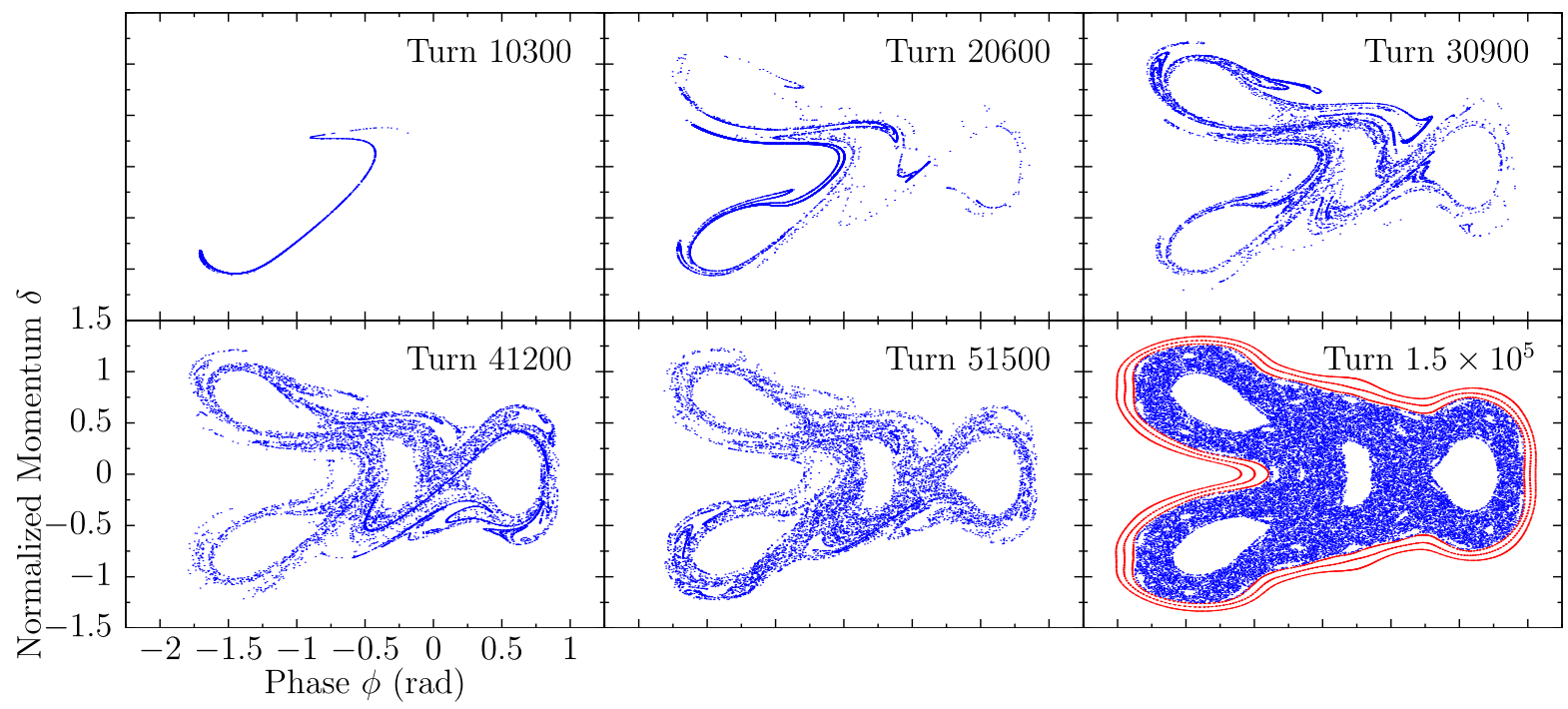

FIG. 10. The evolution of the small bunch of $1 \times 10^{4}$ particles with initial rms length $\sigma_{\phi}=1 \times 10^{-3}$ rad and momentum spread $\sigma_{\delta}=1 \times 10^{-3}$ as it undergoes the diffusion process where $r=1 / 2, h=2, a_{m}=58^{\circ}$, and $\Delta \phi_{o}=30^{\circ}$. Particles are observed to be smoothly streaming about the $3: 1$ resonant islands as the diffusion evolves until equilibration at about $1.5 \times 10^{5}$ revolutions. The red points represent particle tracking in the well-behaved bounding tori region. 


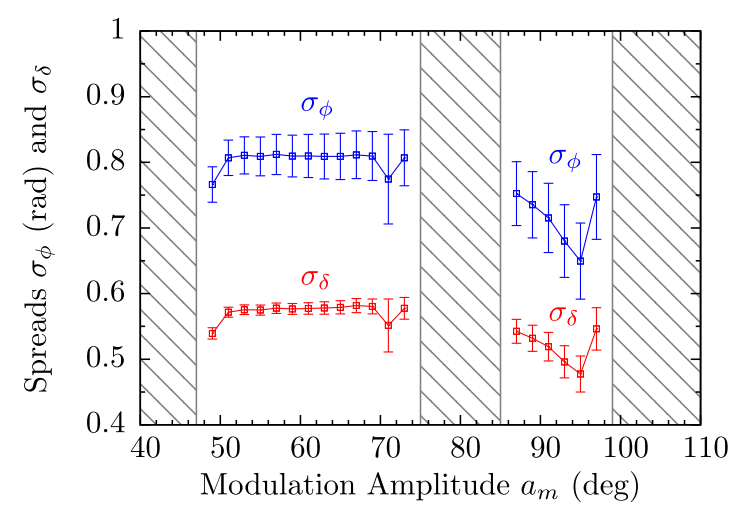

FIG. 11. The rms bunch spreads $\sigma_{\phi}$ and $\sigma_{\delta}$ as a function of modulation amplitude $a_{m}$, where $\Delta \phi_{o}=30^{\circ}$. The hatched region depicts the approximate regions where no diffusion was observed. Beyond $a_{m} \sim 115^{\circ}$, particle loss occurs.

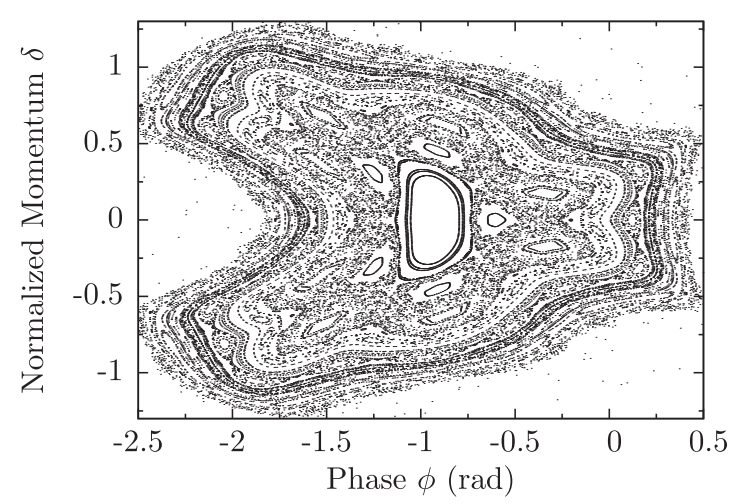

FIG. 12. Nondiffusive Poincaré surface of section depicting the longitudinal phase-space structure on the verge of particle loss where $a_{m}=104^{\circ}$ and $\Delta \phi_{o}=30^{\circ}$. Note that instead of the wellbehaved tori, the structure is bounded by layers of overlapping resonances that will result in particle loss.

amplitude $a_{m}$ parameter space. This will be used to find parameters exhibiting optimal particle bunch diffusion with equilibrium stability. The rapid diffusion of particles typically equilibrates after about $1 \times 10^{5}$ revolutions when the appropriate parameters are chosen. The rms bunch length and momentum spreads were calculated for $1 \times 10^{4}$ macroparticles after $5 \times 10^{5}$ revolutions to ensure that only conditions leading to bounded and lossless diffusion remained in the analysis. The thresholds for maximum rms bunch length and rms momentum spread were based on design limitation such as bunch broadening that fills less than $75 \%$ of a given ring with $h_{1}=1$ to allow for beam extraction.

A map of the rms bunch length in the $a_{m}$ and $\nu_{m} / \nu_{s}$ parameter space for $\Delta \phi_{o}=30^{\circ}$ is shown in Fig. 13. The dark blue regions in the parameter space represent bunch sizes that remained small after $5 \times 10^{5}$ revolutions, i.e., particle bunches that have not undergone diffusion, and dark red regions represent bunch sizes that exceed an upper operable threshold in which particle loss has occurred. It is in between these two parameter limits in which bounded diffusion can be attained.

Referring back to Fig. 5, each normalized synchrotron tune $\frac{n}{M} \times Q_{s} / \nu_{s}$ curve exhibits a negative cubiclike behavior with a minimum near $J \approx 0.4$ and maximum near $J \approx 1.5$. A fixed $\nu_{m} / \nu_{s}$ dotted line may intercept this curve at three points, generating three resonant island chains. If the first two interception points are close to the potential-well minimum, the corresponding two resonant island chains will overlap and become chaotic when the modulation amplitude $a_{m}$ exceeds a certain threshold. Diffusion of the initial bunch will occur when it is close enough to these island chains of overlapped resonances. If the last two interception points are close to the maximum, these two corresponding resonant island chains will overlap, and may lead to chaoticity extending towards the edges of the rf bucket.

For small values of the modulation fraction, e.g., $\nu_{m} / \nu_{s} \sim 1$ or smaller, the relevant $\frac{n}{M} \times Q_{s} / \nu_{s}$ curve (intercepting with the $\frac{n}{M} \lesssim 1$ ) is relatively flat. The minimum does not differ much from the maximum of the curve. The implication is that all three interception points may be close to the minimum and maximum at the same time. Thus with sufficient modulation amplitude $a_{m}$, the nearby island chains will overlap and chaotic regions will be generated from $J \lesssim 0.4$ towards the edges of the rf bucket. This is especially true because the intercepts take place at relatively small gradients $\left|d\left(\frac{n}{M} \times Q_{s} / \nu_{s}\right) / d J\right|$, enhancing the widths of the resonant island chains. Since the bunch is initially far from the minima of all nearby $\frac{n}{M} \times Q_{s} / \nu_{s}$ curves, the bunch particles revolve along well-behaved tori about the potential-well bottom and the bunch is therefore not broadened. As the modulation amplitude increases to $a_{m} \gtrsim 20^{\circ}$, resonant island chains near the three interception points overlap. Chaotic regions are formed and extend to the entire phase space. This explains why the initial bunch rapidly diffuses to fill the entire phase space resulting in particle loss instead of bounded diffusion.

When the modulation fraction increases, for example, to $\nu_{m} / \nu_{s} \sim 1.5$ to 2 , the relevant $\frac{n}{M} \times Q_{s} / \nu_{s}$ curves exhibit large normalized tune differences between the minima and maxima. It then becomes impossible for the three interception points to be close to both the minimum and maximum of the intercepted $\frac{n}{M} \times Q_{s} / \nu_{s}$ curve. This implies that the chaotic region generated by resonant island chains near the maxima of the relevant $\frac{n}{M} \times Q_{s} / \nu_{s}$ curves (outer chaotic region) will be separated from the chaotic region generated by overlapping resonant island chains near the minima (inner chaotic region) by the well-behaved tori. Thus the initial bunch will diffuse into this inner chaotic region when $a_{m}$ is sufficiently large, but bounded by wellbehaved tori so that particles will not diffuse into the outer chaotic region where it would be lost. This explains the existence of the large region of parameter space resulting in 


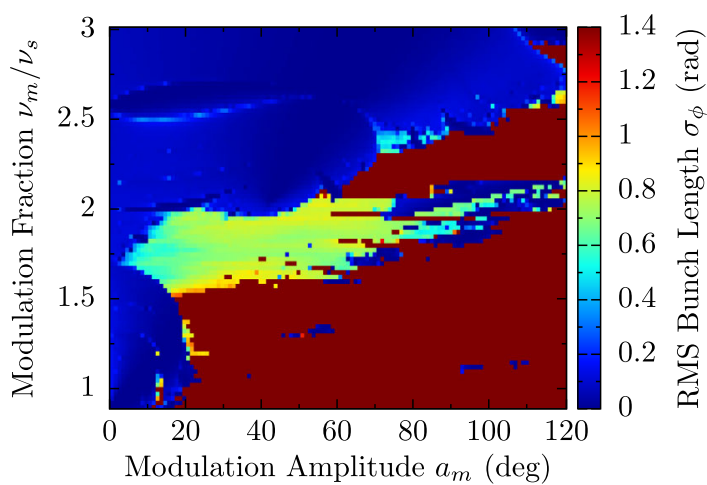

FIG. 13. Parameter map of the rms bunch length $\sigma_{\phi}$ as a function of the modulation amplitude $a_{m}$ and modulation fraction $\nu_{m} / \nu_{s}$ at $\Delta \phi_{o}=30^{\circ}$. The dark blue regions represent parameters where the particles are confined and no diffusion occurs. The dark red regions represent parameters where the diffusion is unbounded and particles are lost.

bounded particle diffusion, as shown in Fig. 13, when the modulation fraction is between $\nu_{m} / \nu_{s} \sim 1.5$ and 2 .

As the modulation fraction increases further from $\nu_{m} / \nu_{s} \sim 2$ to 3 , the fixed-value $\nu_{m} / \nu_{s}$ horizontal line will intercept with each relevant $\frac{n}{M} \times Q_{s} / \nu_{s}$ curve at much larger slopes $\left|d\left(\frac{n}{M} \times Q_{s} / \nu_{s}\right) / d J\right|$ than when $\nu_{m} / \nu_{s}$ is small. The width of the corresponding resonant island chains becomes narrower. Thus island chains will not overlap as easily, and chaotic regions will be much more difficult to generate. For this reason, diffusion of the bunch can hardly occur at large modulation fractions.

In the modulation fraction range $1.6 \lesssim \nu_{m} / \nu_{s} \lesssim 2.0$, Fig. 13 shows a relatively sizable parameter region with bounded particle diffusion for $\Delta \phi_{o}=30^{\circ}$. The rms bunch lengths in this parameter region are in the range $0.5 \mathrm{rad} \lesssim$ $\sigma_{\phi} \lesssim 0.9 \mathrm{rad}$ and $\mathrm{rms}$ momentum spread in the range $0.4 \lesssim \sigma_{\delta} \lesssim 0.6$. The parameter insensitivity of the applied phase modulation in the beam dilution technique indicates that desired diluted results can be readily obtained without impractical and prohibitively fine-tuning capabilities of the rf control system.

\section{C. $r f$ phase difference $\Delta \phi_{o}=45^{\circ}$}

Although the rf phase difference $\Delta \phi_{o}=30^{\circ}$ provides the maximum shift of the rf potential-well bottom from the origin of the primary-rf-only phase space, this scenario does have some shortcomings. According to Figs. 4 and 5, the intercepts of the $\nu_{m} / \nu_{s}=2$ horizontal dotted line with the $3 \times Q_{s} / \nu_{s}$ resonance curve are far away from the initial location of the particle bunch, implying that the bunch is initially far from the fixed points of the first-order $3: 1$ resonance. This limits the bunch from falling inside the stochastic layers surrounding the separatrices unless the modulation amplitude $a_{m}$ is sufficiently large. In addition, Fig. 6 shows that the resonance strength function of the $3: 1$ resonance is much smaller than that of the $2: 1$ resonance.

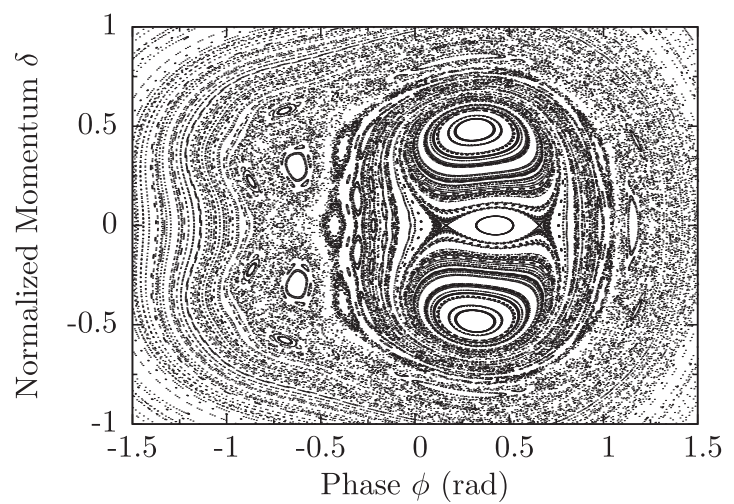

FIG. 14. Nondiffusive Poincaré surface of section depicting the longitudinal phase-space structure with parameters $a_{m}=7^{\circ}$, $\nu_{m} / \nu_{s}=2$, and $\Delta \phi_{o}=45^{\circ}$. The resonant islands pertaining to the $2: 1$ resonance can be seen near the primary-rf-only phasespace origin.

As a result, relatively large modulation amplitude is necessary to drive the bunch into diffusion. These shortcomings motivated the examination of another rf phase difference $\Delta \phi_{o}=45^{\circ}$, corresponding to the potential-well bottom offset of $\phi_{0}=29.12^{\circ}$ which is only $3 \%$ less than the maximum potential-well bottom offset when $\Delta \phi_{o}=30^{\circ}$.

For $\Delta \phi_{o}=45^{\circ}$, Fig. 15 shows the $2 \times Q_{s} / \nu_{s}$ curve shifted up to intercept the $\nu_{m} / \nu_{s}=2$ horizontal dotted line. With the boxed interception points occurring at approximately $J=0.085$, the $2: 1$ resonant islands, and more importantly, the unstable fixed points of the $2: 1$ resonance are now much closer to the initial location of the bunch at $J=0.106$. Now relatively smaller modulation amplitudes are expected for particle diffusion to occur. The resonance strengths $\left|g_{n}(J)\right|$ for $\Delta \phi_{o}=45^{\circ}$, shown in

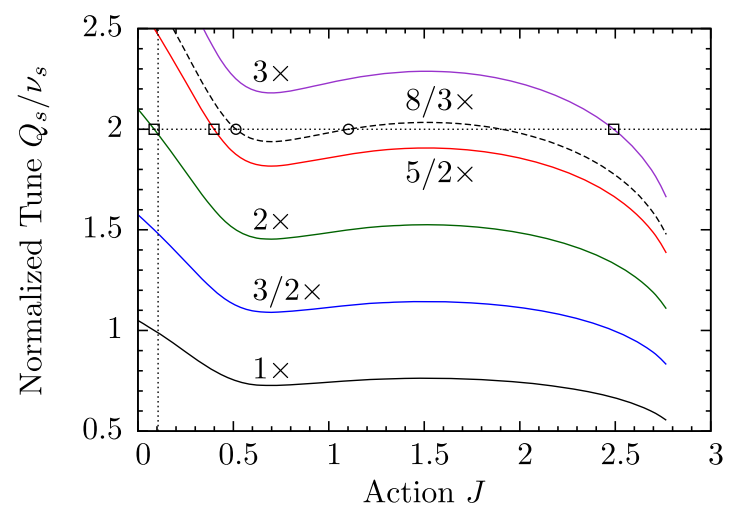

FIG. 15. Normalized synchrotron tune $Q_{s} / \nu_{s}$ as functions of the action $J$. The $\nu_{m} / \nu_{s}=2$ horizontal dotted-line interception illustrates the parametric resonances driven by phase modulation where the double rf parameters are $r=1 / 2, h=2$, and $\Delta \phi_{o}=45^{\circ}$. The vertical dotted line where $J=0.106$ depicts the initial location of the bunch at the origin of the primary-rfonly phase space. The circular interception points depict the two relevant $8: 3$ resonances that are required to be disrupted to enable a larger chaotic region. 


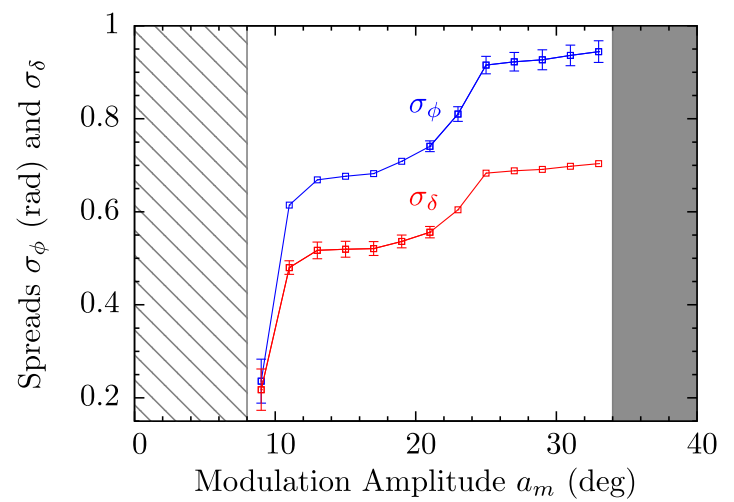

FIG. 16. The rms bunch spreads $\sigma_{\phi}$ and $\sigma_{\delta}$ as functions of modulation amplitude $a_{m}$ where $\Delta \phi_{o}=45^{\circ}$. The hatched region depicts the approximate regions where no diffusion was observed. The solid band depicts the approximate region where beam loss was encountered. Data points where the error is much less than $1 \%$ have negligible error bars.

Fig. 6, were found to deviate only slightly from the case when $\Delta \phi_{o}=30^{\circ}$ with no other unique features to be noted.

The nondiffusive Poincaré section when $a_{m}=7^{\circ}$ and $\Delta \phi_{o}=45^{\circ}$ is illustrated in Fig. 14. Immediately, the 2:1 resonance structure can be identified near the origin of the primary-rf-only phase space, as predicted. The small bunch, however, will remain confined to the thin layer higher-order resonances surrounding the $2: 1$ resonant islands and cannot be driven to the larger chaotic regions formed by the overlap of the $7: 3,8: 3$ and higher-order resonances beyond. For the bunch to escape and diffuse, the thin higher-order chains of islands enclosing the 2:1 resonance structure must be disrupted and collapse. The sharp increase in the rms bunch size $\sigma_{\phi}$ and $\sigma_{\delta}$, shown in Fig. 16, was observed when $a_{m} \approx 9^{\circ}$. This marks the condition where the thin bounding higher-order chains of resonant islands have successfully collapsed to allow for the diffusion of the small particle bunch.

With the modulation amplitude increased just beyond $a_{m} \approx 9^{\circ}$, the bunch size briefly levels off and then increases again for the second time at $a_{m} \approx 23^{\circ}$. This second sudden increase of the $\sigma_{\phi}$ and $\sigma_{\delta}$ can be explained by the bifurcation of multiple $8: 3$ resonances, each with its own set of resonant islands. Their individual positions in action space are depicted as circles in Fig. 15. The first set of resonant islands associated with the 8:3 resonance nearest to $J=0$ collapses resulting in the first sharp increase in the bunch size when $a_{m} \approx 9^{\circ}$. As the modulation amplitude increases to $a_{m} \approx 23^{\circ}$, the collapse of the second set of $8: 3$ resonant islands results in the sharp increase in the bunch size for the second time. The Poincaré section in Fig. 17 depicts the condition just before the second set of $8: 3$ resonant island chain shrinks and collapses. The chain of eight resonance islands associated with the second $8: 3$ resonance can be seen surrounding the entire phase-space structure. The remnants of both sets of the $8: 3$ resonant

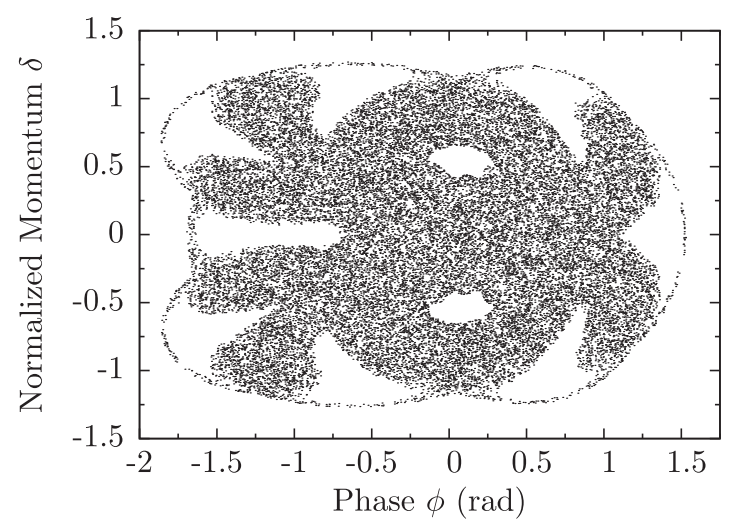

FIG. 17. Poincaré surface of section depicting the diffusion of a small bunch where $a_{m}=23^{\circ}$ and $\Delta \phi_{o}=45^{\circ}$ just as the bunch sizes sharply increase for the second time. Note the islands of the $8: 3$ resonance at the edge of the diffused structure just before collapse and subsequent shrinking.

islands, after the modulation amplitude has been increased beyond $a_{m}=23^{\circ}$, can be readily identified in Fig. 18 as small empty voids in an otherwise homogeneous structure.

The Poincare section where $a_{m}=28^{\circ}$ and $\Delta \phi_{o}=45^{\circ}$ is shown in Fig. 18. As compared to Fig. 10, the large voids of the $3: 1$ resonance stable fixed points are now replaced by smaller voids, pertaining to the stable fixed points of the $2: 1$ and $8: 3$ resonance. These resonant islands are now completely enveloped by the thick layer of chaos. The overall phase-space structure can be seen to be broader and more rectangular as compared to the case where $\Delta \phi_{o}=30^{\circ}$. The rms bunch length and momentum spreads, calculated after equilibration, are $\sigma_{\phi}=0.93 \pm 0.02 \mathrm{rad}$ and $\sigma_{\delta}=0.689 \pm 0.004$.

The choice of these parameters provides a greater degree of longitudinal particle distribution uniformity, as shown in

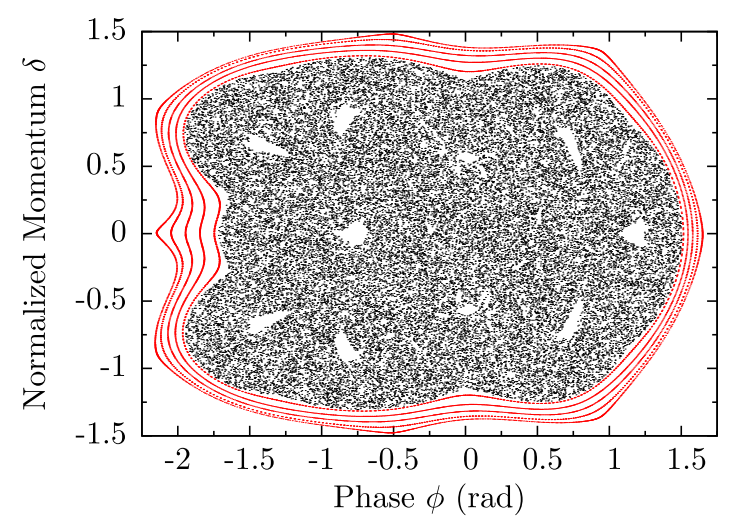

FIG. 18. Black: Poincaré surface of section of a small Gaussian bunch of rms length $\sigma_{\phi}=1 \times 10^{-3} \mathrm{rad}$ and momentum spread $\sigma_{\delta}=1 \times 10^{-3}$ initially at the phase-space center at the last modulation periods of 1.5 million revolutions where $a_{m}=28^{\circ}$ and $\Delta \phi_{o}=45^{\circ}$. The red points depict single-particle tracking of test particles placed initially near the edge of the phase-space structure. 


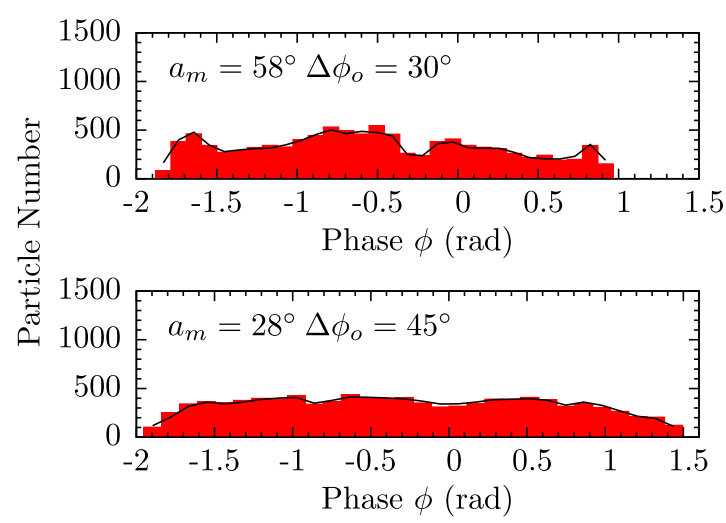

FIG. 19. Histogram of 30 bins spanning the entire length comparing the linear particle distribution of the two different double rf settings after diffusion. The black line depicts the turnby-turn averages of the individual bins $1 \times 10^{6}$ revolutions after diffusion, confirming the stability of the structure.

Fig. 19. Furthermore, the diffusion process converges about twice as quickly, after $6.5 \times 10^{4}$ revolutions shown in Fig. 20, as opposed to $1.5 \times 10^{5}$ revolutions. These improvements were attained at $a_{m}=28^{\circ}$, about half the modulation amplitude compared to the dilution case where $\Delta \phi_{o}=30^{\circ}$. The parameter map of the rms bunch lengths in the $a_{m}$ and $\nu_{m} / \nu_{s}$ parameter space for $\Delta \phi_{o}=45^{\circ}$, shown in Fig. 21, depicts a similar parameter region for beam dilution as compared to Fig. 13 where $\Delta \phi_{o}=30^{\circ}$. The parameter map of the rms momentum spread exhibits the same features as Fig. 21 with range $0.5 \lesssim \sigma_{\delta} \lesssim 0.7$ observed for bunch dilution. The sizable operable region of parameter space ensures beam dilution will be observed in experiments without unnecessarily strict constrains on operating parameters and rf control systems.

\section{Effects of radiation damping}

When radiation damping occurs at a much longer time scale as compared to the rapid particle diffusion, the effects

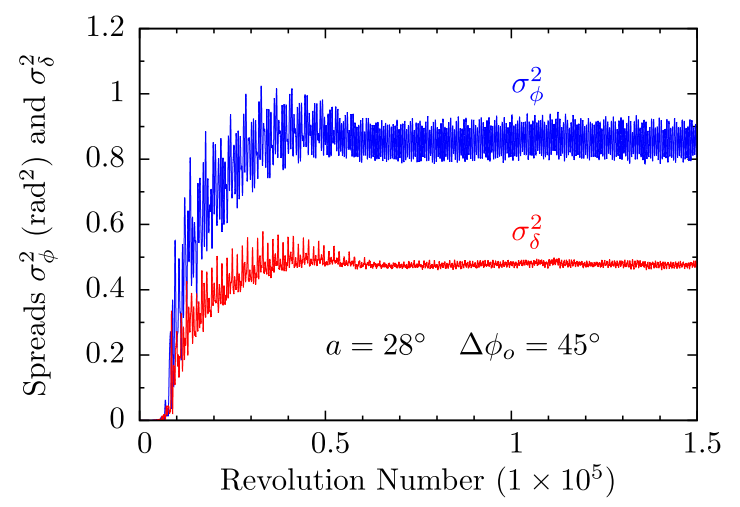

FIG. 20. Longitudinal bunch sizes as functions of revolution number for $a_{m}=28^{\circ}$ and $\Delta \phi_{o}=45^{\circ}$ as the diffusion process evolves and equilibrates after about $6.5 \times 10^{4}$ revolutions.

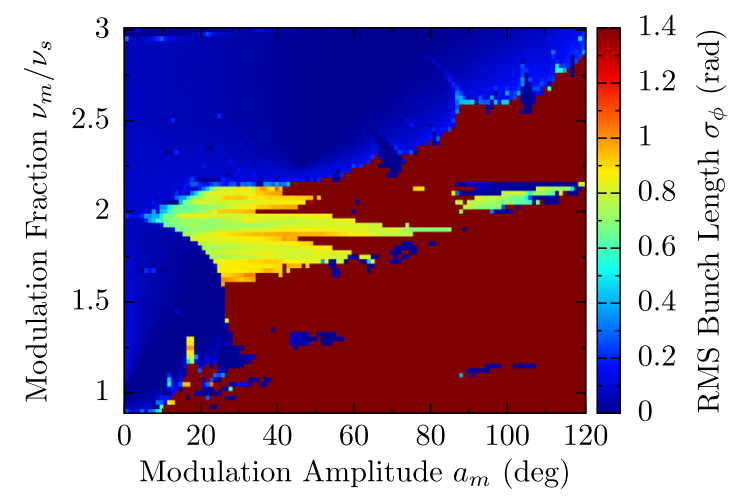

FIG. 21. Parameter map of the rms bunch length as a function of the modulation amplitude and modulation fraction at $\Delta \phi_{o}=45^{\circ}$. The dark blue regions represent particle confinement where no diffusion occurs. The dark red regions represent diffusion occurrence, where the diffusion is unbounded with particle loss.

of radiation damping can be neglected in the theoretical analysis and numerical simulations [8]. The particle motion then becomes Hamiltonian, which facilitates analysis, e.g., through the introduction of action-angle variables. Nevertheless, radiation damping can be important in certain cases. To incorporate the effects of radiation damping in the investigation, Eq. (20) has been modified to

$$
\delta_{k+1}=\delta_{k} e^{-T_{o} / \tau_{\|}}-2 \pi \nu_{s}\left[\sin \phi_{k+1}-r \sin \left(h \phi_{k+1}+\Delta \phi_{o}\right)\right],
$$

where $\tau_{\|}$is the radiation damping time of the longitudinal emittance and $T_{o}$ is the period of one revolution. This modification constitutes an overestimation of the damping effects as random quantum excitations have not been included. We can continue to neglect quantum excitations as the phase-space area of the chaotic region in which particles diffuse into is much larger than the area of the initial radiation-damped bunch. For the $20 \mathrm{~m}$ compact electron storage ring at Indiana University, the longitudinal emittance damping time is of the order of seconds, due to the low operating energy of $\sim 50 \mathrm{MeV}$. On the other hand, simulation results that are shown in Fig. 20 where $a_{m}=28^{\circ}$ and $\Delta \phi_{o}=45^{\circ}$, indicate that it takes about $n_{\text {req }} \sim 5 \times 10^{4}$ revolutions for the bunch diffusion to reach equilibrium. Thus radiation damping plays no role in the beam dilution process for this storage ring.

According to the derivation given in Sec. II, the smallamplitude synchrotron tune $\nu_{s}$ in the absence of the higher harmonic rf system is an important parameter in the rate at which particles diffuse towards the chaotic regions for beam dilution. The number of required revolutions to reach particle diffusion equilibrium is inversely proportional to $\nu_{s}$. Thus, for any electron ring with a secondary rf cavity, phase difference of $\Delta \phi_{o}=45^{\circ}$, and with small-amplitude synchrotron tune $\nu_{s}$ in the presence of only the primary $\mathrm{rf}$ 
TABLE I. Properties of various electron or positron rings, ALS at LBL, BEPC at IHEP, LER and HER of PEP II at SLAC, CESR at Cornell, APS at ANL, and LEP at CERN, showing in particular the longitudinal damping time $\tau_{\|}$, number of revolutions required for emittance blowup via diffusion $n_{\text {req }}=T_{\text {req }} / T_{o}$, and ratio $\tau_{\|} / T_{\text {req }}$.

\begin{tabular}{lcccccccc}
\hline \hline & ALPHA & LER & CESR & BEPC & HER & ALS & LEP & APS \\
\hline Beam energy $(\mathrm{GeV})$ & 0.05 & 3.2 & 6 & 2.2 & 9 & 1.5 & 55 & 7 \\
Circumference $(\mathrm{m})$ & 20 & 2199 & 768.4 & 240.4 & 2199 & 196.8 & 26659 & 1104 \\
Revolution period $T_{o}(\mu \mathrm{s})$ & 0.067 & 7.34 & 2.563 & 0.802 & 7.34 & 0.656 & 88.93 & 3.683 \\
Synchrotron tune $\nu_{s}$ & 0.00097 & 0.034 & 0.064 & 0.016 & 0.0522 & 0.0082 & 0.085 & 0.0061 \\
Longitudinal damping time $\tau_{\|}(\mathrm{ms})$ & 10000 & 77 & 8.0 & 8.8 & 19 & 8.8 & 19 & 4.7 \\
$n_{\|}($revolutions) & $1.50 \times 10^{8}$ & 10500 & 3120 & 11000 & 2590 & 13400 & 214 & 1280 \\
Revolution required for emittance & 50000 & 1430 & 758 & 3030 & 930 & 5920 & 571 & 7960 \\
$\quad$ blowup $n_{\text {req }}=T_{\text {req }} / T_{o}$ & & & & & & & & \\
$\tau_{\|} / T_{s}\left(10^{3}\right)$ & 154000 & 309 & 48.7 & 686 & 50 & 1640 & 2.52 & 209 \\
$\tau_{\|} / T_{\text {req }}\left(=n_{\|} / n_{\text {req }}\right)$ & 2998 & 7.35 & 4.11 & 3.62 & 2.78 & 2.27 & 0.375 & 0.161 \\
\hline \hline
\end{tabular}

cavity, the number of revolution to reach equilibrium is approximately given by

$$
n_{\text {req }} \nu_{s}=\left(n_{\text {req }} \nu_{s}\right)_{\mathrm{ALPHA}},
$$

where for the electron storage ring at IU, $\left(n_{\text {req }}\right)_{\mathrm{ALPHA}} \approx$ $5 \times 10^{4}$ and $\left(\nu_{s}\right)_{\mathrm{ALPHA}}=1 / 1030$. The time to reach blowup equilibrium is therefore $T_{\text {req }}=n_{\text {req }} T_{o}$, where $T_{o}$ is the revolution period of the storage ring under consideration. For ALPHA, $T_{\text {req }} \sim 3.3 \mathrm{~ms}$.

The longitudinal damping times of some electron rings are tabulated in Table I. With the exception of the ALPHA storage ring, the longitudinal damping times range from $\tau_{\|}=4.7$ to $77 \mathrm{~ms}$. These damping times should be compared with the corresponding time $T_{\text {req }}$ to reach emittance blowup. The effects of radiation damping will not be important when $\tau_{\|} / T_{\text {req }} \gtrsim 1$ or $n_{\|} / n_{\text {req }} \gtrsim 1$. This is true for the lower-energy rings like the ALS at LBL, BEPC at IHEP, LER and HER of PEP II at SLAC.

Next the effects on radiation damping on the beam diffusion process when $\tau_{\|} / T_{\text {req }} \leq 1\left(n_{\|} / n_{\text {req }} \leq 1\right)$ will be investigated. Continuing the simulations with electron storage ring at IU where the rf phase difference $\Delta \phi_{o}=$ $45^{\circ}$ and phase-modulation amplitude $a_{m}=28^{\circ}$, but with finite longitudinal damping time reduced to $n_{\|}=5 \times 10^{4}$ revolutions. The top row of the Poincaré sections, depicted in Fig. 22, shows the evolution of particle diffusion after $n_{\text {req }}=5 \times 10^{4}$ revolutions and the bottom row shows $2 n_{\text {req }}=1 \times 10^{5}$ revolutions for various values of $n_{\|} / n_{\text {req }}$. The amount of radiation damping is characterized by $n_{\|} / n_{\text {req }}$. These Poincaré sections are to be compared to those in Fig. 18, where radiation damping was not included $\left(n_{\|}=\infty\right)$ in the simulations. The effect of radiation damping is easily observed in that the diffused bunch occupies a

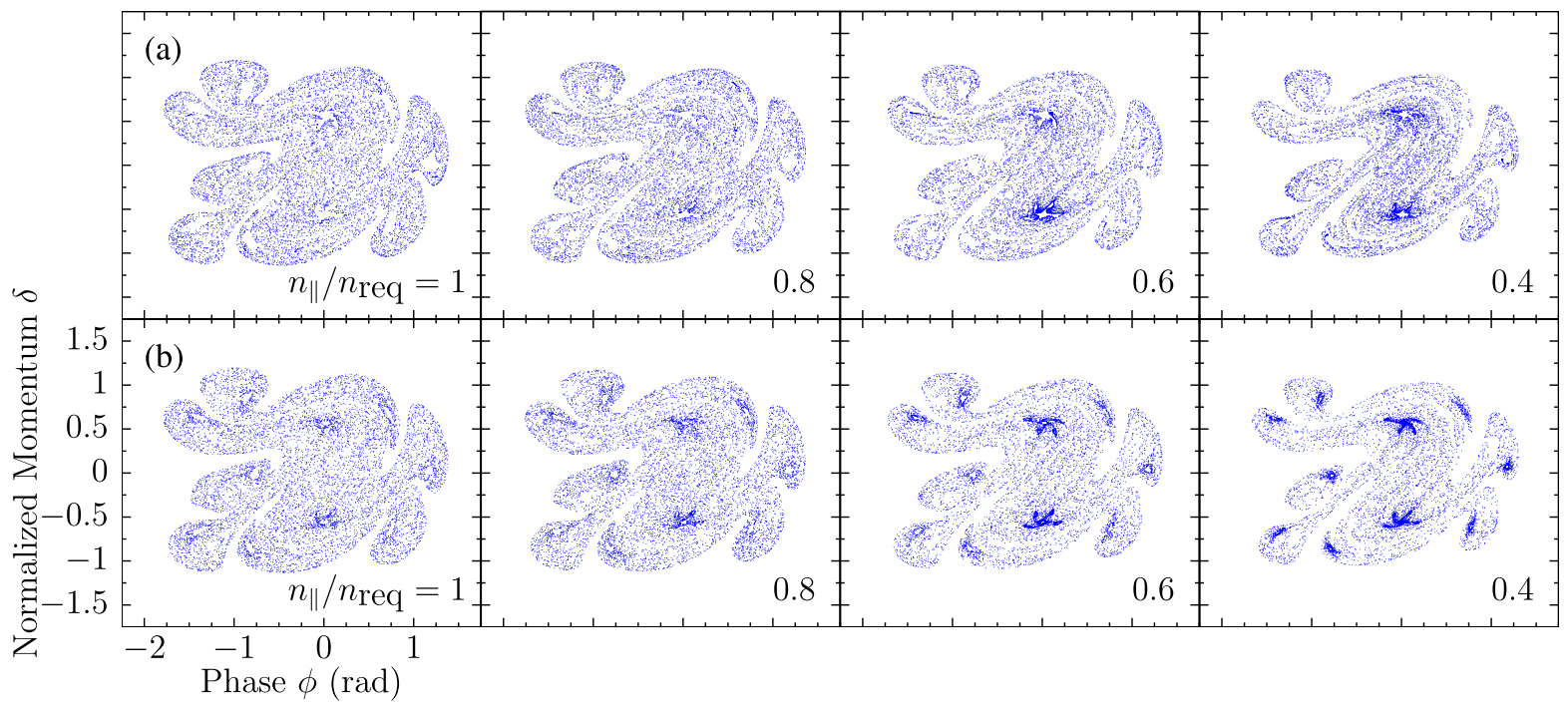

FIG. 22. Poincaré surfaces of section where $a_{m}=28^{\circ}, \Delta \phi_{o}=45^{\circ}, \nu_{m}=2 \nu_{s}, r h=1$, and radiation damping considered as opposed to the case in Fig. 18 where radiation damping has been neglected. The top row (a) corresponds to a tracking simulation where $n_{\text {req }}=$ 50,000 revolutions and the bottom row (b) $2 n_{\text {req }}=100,000$ revolutions with the columns $n_{\|} / n_{\text {req }}=1,0.8,0.6$, and 0.4 , respectively. As the radiation-damping time decreases and as the viewing time increases, the general trend is the continual shrinking of the stable diffused bunch area with more empty spaces and more particles spiraling into the attractors. 
smaller area in phase space and exhibits more empty regions. Nevertheless, the small bunch is broadened, is still bounded, and maintains uniform longitudinal density as required by the radiation-effects experiments. It is to be noted that there are also a number of small empty regions in Fig. 18. Each of them, in fact, contains a stable fixed point. In the presence of radiation damping during the dilution process, these stable fixed points become attractors in which particles are drawn towards. This explains the areas of higher particle density about these stable fixed points observed in the Poincare sections on the bottom row of Fig. 22 where particle tracking was carried out for 100,000 revolutions.

The longitudinal damping time is lowered to $n_{\|}=40,000$ revolutions corresponding to $n_{\|} / n_{\text {req }}=0.8$. The resulting Poincaré sections after $n_{\text {req }}=5 \times 10^{4}$ and $2 n_{\text {req }}=1 \times 10^{5}$ revolutions are depicted in the second column of Fig. 22. The third and fourth columns show similar simulation results with $n_{\|}=3 \times 10^{4}$ revolutions $\left(n_{\|} / n_{\text {req }}=0.6\right)$ and $n_{\|}=$ $2 \times 10^{4}$ revolutions $\left(n_{\|} / n_{\text {req }}=0.4\right)$, respectively. A trend in the shrinking of the phase-space area occupied by the diffused particle bunch is observed with particles being drawn towards the attractors. Two conclusions can be drawn. First, this diffusion process of bunch broadening with uniform longitudinal distribution uniformity is possible only when $n_{\|} / n_{\text {req }} \gtrsim 1$, where the number of required revolutions $n_{\text {req }}$ is calculated according to Eq. (22). Second, the equilibrium diffused particle bunch must be extracted before $\sim 2 n_{\|}$revolutions otherwise particles will eventually migrate towards the attractors preventing the condition of longitudinal particle distribution uniformity.

\section{E. Discussion of beam dilution parameters}

There are many parameters in this applied phase modulation beam dilution model. Besides the phase-modulation amplitude $a_{m}$, the other parameters are the rf harmonic ratio $h=h_{2} / h_{1}$, the rf voltage ratio $r=V_{2} / V_{1}$, the modulation tune fraction $\nu_{m} / \nu_{s}$, the rf phase difference $\Delta \phi_{o}$, and the modulation phase $\eta$. To reduce the number of parameters, the constraint $r h=1$ was introduced for the analysis. Choice of various beam dilution parameters are discussed and summarized as follow:

1. It is necessary to shift the rf potential-well bottom as far as possible from the origin of the primary-rf-only longitudinal phase space at $\phi=0$, so that the strengths of parametric resonances will be optimally large. The maximum shift, $\phi_{o}=\sin ^{-1} r$, is independent of the $\mathrm{rf}$ harmonic ratio $h$. Since $h$ must be an integer larger than one for the secondary rf cavity to form the higher-harmonic cavity system and $r$ must not be too small as to maximize potential-well offset $\phi_{o}$, with the restriction $r h=1$, it has been shown that the choice $h=2$ would lead to an optimally large $r$. Therefore the largest $\phi_{o}$ can be obtained so that parametric resonances driven by the applied phase modulation would become most efficient.
2. The rf phase difference $\Delta \phi_{o}$ for the maximum shift of the rf potential-well bottom from $\phi=0$ is $\Delta \phi_{o}=$ $\pi / 2-h \sin ^{-1} r$. For small values of $r$, the phase difference is approximately $\Delta \phi_{o}=30^{\circ}$. As shown in Fig. 2, however, shifting of the rf potential-well bottom $\phi_{o}$ is not very sensitive for values of $\Delta \phi_{o}$ about $\Delta \phi_{o}=30^{\circ}$. The choice of $\Delta \phi_{o}=45^{\circ}$ rather than the ideal $30^{\circ}$ will reduce the maximum shift by only $3 \%$. As a result, the choice of $\Delta \phi_{o}$ can be varied so as to allow the small particle bunch initially located at $\phi=0$ to be closest to a chains of overlapping resonant islands that strongly excite the particle motions to drive bunch diffusion.

3. The choice of the modulation fraction $\nu_{m} / \nu_{s}$ was found to be between $\nu_{m} / \nu_{s} \sim 1.6$ and $\nu_{m} / \nu_{s} \sim 2$ for optimal rapid particle diffusion to occur. This was described in detail near the end of Sec. III B where the map of the relevant parameter space shown in Figs. 13 and 21 was introduced. 4. The longitudinal phase-space structure rotates in phase space after every subsequent revolution. To avoid the confusing effect of this rotation, stroboscopic frames at every $1 / \nu_{m}$ revolutions were depicted. When a nonvanishing modulation phase $\eta \neq 0$ is chosen, the stroboscopic Poincaré section will also rotate about the potentialwell minimum $\phi_{o}$ as compared to the case where $\eta=0$. Due to the differences in the synchrotron rotational frequency at various actions or distances from the $\mathrm{rf}$ potential-well bottom, the Poincaré section at various instants in the rotation for $\eta \neq 0$ will differ from pure rotation where $\eta=0$. This will cause the phase-space structure to twist and be distorted.

The dilution model has also been studied with relaxation of the restriction $r h=1$. However, the lengthy and similar results will not be reported.

\section{IMPLEMENTATION}

The Advanced Electron Photon Facility (ALPHA) is a joint collaboration between the Indiana University Center for Exploration of Energy and Matter (CEEM) and the Crane Naval Surface Warfare Center [1], designed for extreme environment radiation-effects experiments. The $20 \mathrm{~m}$ circumference electron storage ring, designed with betatron tunes of $\nu_{x}=1.75$ and $\nu_{z}=0.75$, will be injected with electrons at full energy from a $50-100 \mathrm{MeV}$ linac source. The gradient damping wigglers pair enables variability of the momentum-compaction factor for storage ring operations in the isochronous condition.

The project requires a single $50-100 \mathrm{MeV}$ electron bunch to be broadened up to $40 \mathrm{~ns}$ with uniform longitudinal particle distribution. Electrons are introduced into the storage ring through a series of multiturn injections, utilizing closed-orbit distortions, for charge accumulation to attain a total bunch charge of up to $600 \mathrm{nC}$. The stored bunch then undergoes radiation damping to reach an equilibrium bunch length of 10-100 ps. Beam dilution is initiated by activating the secondary rf cavity in conjunction with rf 
phase modulation with chosen parameters $a_{m}$ and $\Delta \phi_{o}$ applied to both rf cavities. After the dilution process have equilibrated, the broadened bunch is ready to be extracted. A traveling-wave fast-extraction kicker will divert the beam through a Lambertson septum into the extraction line where third-order focusing with an octupole magnet pair will provide nonlinear beam spreading before the beam impinges on a target. The resulting beam will have longitudinal particle distribution uniformity with minimal secondary effects at the device under test. Due to the size of the compact storage ring and requirements of the radiationeffects experiments, the beam dilution method proposed will be an ideal candidate for the task.

The electron storage ring is currently under construction. A compact ferrite-loaded quarter-wave $15 \mathrm{MHz}$ rf cavity [14] has been designed, fabricated, and commissioned for the electron storage ring. The design of the second rf cavity operating at $30 \mathrm{MHz}$ and the low-level rf controls to facilitate the beam dilution technique are currently being carried out and awaiting final approval for construction. The implementation of phase-space beam dilution method using an ac dipole has been proposed to simplify the application of beam dilution. This study will be presented at a later time.

\section{CONCLUSION}

A method of phase-space beam dilution has been presented for the broadening and longitudinal uniformization of a small radiation-damped particle bunch. This is accomplished by applying phase modulation to a double rf system configured for variability in the relative phase between the two rf cavities. Parametric resonances are driven within the particle bunch in the presence of the applied phase modulation. When a multitude of these resonances combine and overlap, large regions of chaotic particle motion are generated. The size and effectiveness of various chaotic regions for bounded particle diffusion are related to the dilution properties that include the resonance strengths, topologies of resonant islands driven within the bunch, initial location of the small bunch, and existence of the well-behaved bounding tori. These properties are determined by the important rf parameters that include the phase difference parameter $\Delta \phi_{o}$, modulation amplitude $a_{m}$, and modulation tune fraction $\nu_{m} / \nu_{s}$.

The theoretical model for the double rf system has been presented to analyze parametric resonances that arise from the applied phase modulation. Symplectic multiparticle tracking simulation results have been presented to characterize phase modulation driven parametric resonant structures and find resonant island topologies that promote particle bunch dilution. It has been shown that a large parameter region in the $a_{m}$ and $\nu_{m} / \nu_{s}$ parameter space exists where bounded diffusion of a particle bunch was observed. This ensures that the beam dilution method presented can be readily achieved in experimentation without strict tolerances on the if control system. Optimal parameters for phase-space beam dilution for radiationeffects experiments in the compact storage ring at Indiana University have been found.

\section{ACKNOWLEDGMENTS}

We would like to thank members of the Accelerator Physics Group at Indiana University, especially X. Shen, $\mathrm{M}$. Ng, and P. McChesney, for their helpful suggestions and fruitful discussions. This work is supported in part by grants from the U.S. Department of Energy under Contracts No. DE-FG02-12ER41800, No. DE-AC02-76CH030000, and the National Science Foundation (NSF) No. PHY1504778.

[1] S. Y. Lee, J. Kolski, Z. Liu, X. Pang, C. Park, W. Tam, and F. Wang, Low energy electron storage ring with tunable compaction factor, Rev. Sci. Instrum. 78, 075107 (2007); S.-Y. Lee et al., The ALPHA Project at IU CEEM, in Proceedings of the International Particle Accelerator Conference, Kyoto, Japan (ICR, Kyoto, 2010), p. 268.

[2] R. Gram and P. Morton, Advantages of a set of second harmonic rf cavities, Report No. SLAC TN-67-30, 1967; A. Hofmann and S. Meyers, Beam dynamics in a double rf system, in Proceedings, 11th International Conference on High-Energy Accelerators, HEACC, 1980 (CERN, Geneva, Switzerland, 1980), edited by W. S. Newman, p. 610; R. Biscardi and G. Ramirez, RF system for bunch lengthening, in Proceedings of the Particle Accelerator Conference, Dallas, TX, 1995 (IEEE, New York, 1995), p. 1660.

[3] J. M. Baillod, L. Magnani, G. Nassibian, F. Pedersen, and W. Weissflog, A second harmonic (6-16 MHz) rf system with feedback-reduced gap impedance for accelerating flattopped bunches in the CERN PS booster, IEEE Trans. Nucl. Sci. 30, 3499 (1983); G. Gelato, L. Magnani, N. Rasmussen, H. Schönauer, and K. Schindl, Progress in space-charge limited machines: Four times the design intensity in the CERN proton synchrotron booster, in Proceedings of the 1987 Particle Accelerator Conference (IEEE, Washington, DC, 1987).

[4] J. E. Griffin, C. Ankenbrandt, J. A. MacLachlan, and A. Moretti, Isolated bucket rf system in the Fermilab Antiproton Facility, IEEE Trans. Nucl. Sci. 30, 3502 (1983); S. Y. Lee and K. Y. Ng, Particle dynamics in storage rings with barrier rf systems, Phys. Rev. E 55, 5992 (1997); C. M. Bhat, Barrier rf system in synchrotrons, in Proceedings of the 9th European Particle Accelerator Conference, Lucerne, 2004 (EPS-AG, Lucerne, 2004), p. 236.

[5] R. Cappi, R. Garoby, and E. Shaposhnikova, Experimental study of controlled longitudinal blowup, Report No. CERN-PS-92-40-RF, 1992; J. M. Brennan, L. Ahrens, P. Cameron, W. Frey, M. A. Goldman, D. Kasha, J. Kats, A. McNerney, E. Raka, and R. Sanders, A high harmonic cavity for controlled longitudinal phase space dilution in the AGS, in Proceedings of 1st EPAC Conference, Rome, 
Italy, 1988, edited by S. Tazzari, p. 1006; E. Shaposhnikova, Controlled longitudinal emittance blowup in the SPS using a 4th harmonic rf system, Part. Accel. 58, 257 (1997).

[6] G. Papotti, T. Bohl, T. P. R. Linnecar, E. N. Shaposhnikova, and J. Tuckmantel, Study of controlled longitudinal emittance blowup for high intensity LHC beams in the CERN SPS, in Proceedings of the 11th European Particle Accelerator Conference, Genoa, 2008 (EPS-AG, Genoa, Italy, 2008), p. 1676; T. Argyropoulos, T. Bohl, T. P. R. Linnecar, E. N. Shaposhnikova, and J. Tuckmantel, Controlled longitudinal emittance blowup in a double harmonic rf system at CERN SPS, in Proceedings of the 46th ICFA Advanced Beam Dynamics Workshop on High-Intensity and High-Brightness Hadron Beams (HB2010), Morschach, Switzerland, 2010, edited by A. Adelmann, J. Chrin, M. Marx, V. R. W. Schaa, and M. Seidel, p. 86.

[7] T. Toyama, Uniform bunch formation by rf voltage modulation with a band-limited white noise, Nucl. Instrum. Methods Phys. Res., Sect. A 447, 317 (2000); T. Toyama, D. Arakawa, S. Igarashi, J. Kishiro, E. Nakamura, Y. Shimosaki, H. Someya, and K. Takayama, Bunch shaping by rf voltage modulation with a band-limited white signal, KEK Report No. 99-154, 1999.

[8] L. Wang et al., Effect of parametric resonances on the bunched beam dilution mechanism, in Proceedings of the Particle Accelerator Conference, Dallas, TX, 1995, p. 3337; D. Jeon et al., A Mechanism of Anomalous Diffusion in Particle Beams, Phys. Rev. Lett. 80, 2314 (1998).

[9] K. Y. Ng, Particle diffusion in overlapping resonances, in Frascati 1997, Beam dynamics issues for $e^{+} e^{-}$factories, Proceedings of Advanced ICFA Workshop, ICFA'97,
Frascati, Italy, 1997, edited by L. Palumbo and G. Vignola, p. 227; C. M. Chu et al., Effects of overlapping parametric resonances on the particle diffusion process, Phys. Rev. E 60, 6051 (1999).

[10] S. Y. Lee and K. Y. Ng, Application of a localized chaos generated by rf-phase modulations in phase-space dilution, in Proceedings of the 46th ICFA Advanced Beam Dynamics Workshop on High-Intensity and High-Brightness Hadron Beams (HB2010), Morschach, Switzerland, 2010, edited by A. Adelmann, J. Chrin, M. Marx, V. R. W. Schaa, and M. Seidel, p. 639.

[11] J. Y. Liu et al., Analytic solution of particle motion in a double rf system, Part. Accel. 49, 221 (1995).

[12] H. Huang et al., Experimental determination of the Hamiltonian for synchrotron motion with rf phase modulation, Phys. Rev. E 48, 4678 (1993).

[13] $g_{n}(J)$ is calculated by first choosing a $\phi_{1}$ (with $\delta=0$ ) less than the potential-well minimum $\phi_{o}$ as the lower rf phase edge of a torus of motion. The upper end $\phi_{2}$ of the torus is then computed for a given torus of motion. The Hamiltonian value of energy $E$ can next be computed according to Eq. (2) and the momentum offset $\delta$ is known for all rf phases $\phi$ along a given torus. We can then compute the action $J$ of the torus using Eq. (8) and the synchrotron tune $Q_{s}(J)$ using Eq. (14). The angle variable $\psi$ for any rf phase $\phi$ is computed using Eq. (13). Substituting into Eq. (17), the resonant strength $g_{n}(J)$ is obtained.

[14] A. N. Pham, S.-Y. Lee, and T.H. Luo, The ALPHA ferrite-loaded coaxial resonator cavity, in Proceedings of the 3rd International Particle Accelerator Conference, New Orleans, LA, 2012 (IEEE, Piscataway, NJ, 2012), p. 3365 . 